\title{
Correlates of Transitions in Tobacco Product Use by U.S. Adult Tobacco Users between 2013-2014 and 2014-2015: Findings from the PATH Study Wave 1 and Wave 2
}

\author{
Karin A. Kasza ${ }^{1, *}$, Blair Coleman ${ }^{2}$, Eva Sharma ${ }^{3}$, Kevin P. Conway ${ }^{4}$, K. Michael Cummings ${ }^{5}$, \\ Maciej L. Goniewicz ${ }^{1} \oplus$, Raymond S. Niaura ${ }^{6}$, Elizabeth Y. Lambert ${ }^{4}$, Liane M. Schneller ${ }^{1}$, \\ Shari P. Feirman ${ }^{2}$, Elisabeth A. Donaldson ${ }^{2}$, Yu-Ching Cheng ${ }^{2}$, Iilun Murphy ${ }^{2}$, \\ Jennifer L. Pearson ${ }^{6}$, Dennis R. Trinidad ${ }^{7}$, Maansi Bansal-Travers ${ }^{1}$, Tara Elton-Marshall ${ }^{8,9,10,11}$ \\ Daniel A. Gundersen 12,13, Cassandra A. Stanton ${ }^{3}$, David B. Abrams ${ }^{6}$, Geoffrey T. Fong 14,15,16, \\ Nicolette Borek ${ }^{2}$, Wilson M. Compton ${ }^{4}$ and Andrew J. Hyland ${ }^{1}$ \\ 1 Department of Health Behavior, Roswell Park Comprehensive Cancer Center, Buffalo, NY 14263, USA; \\ Maciej.Goniewicz@RoswellPark.org (M.L.G.); Liane.Schneller@RoswellPark.org (L.M.S.); \\ Maansi.Travers@RoswellPark.org (M.B.-T.); Andrew.Hyland@RoswellPark.org (A.J.H.) \\ 2 Office of Science, Center for Tobacco Products, U.S. Food and Drug Administration, Silver Spring, MD 20993, \\ USA; Blair.Coleman@fda.hhs.gov (B.C.); Shari.Feirman@fda.hhs.gov (S.P.F.); \\ Elisabeth.Sherman@fda.hhs.gov (E.A.D.); Yu-Ching.Cheng@fda.hhs.gov (Y.C.-C.); \\ Iilun.Murphy@fda.hhs.gov (I.M.); Nicolette.Borek@fda.hhs.gov (N.B.) \\ 3 Westat, Rockville, MD 20850, USA; EvaSharma@westat.com (E.S.); CassandraStanton@westat.com (C.A.S.) \\ 4 National Institute on Drug Abuse, National Institutes of Health, Bethesda, MD 20892, USA; \\ kconway@rti.org (K.P.C.); elambert@nida.nih.gov (E.Y.L.); wcompton@nida.nih.gov (W.M.C.) \\ 5 Department of Psychiatry and Behavioral Sciences, Medical University of South Carolina, Charleston, \\ SC 29425, USA; cummingk@musc.edu \\ 6 The Schroeder Institute for Tobacco Research and Policy Studies, Truth Initiative, Washington, DC 20001, \\ USA; niaura@nyu.edu (R.S.N.); jennipearson@unr.edu (J.L.P.); david.b.abrams@nyu.edu (D.B.A.) \\ 7 Department of Family Medicine and Public Health, University of California, La Jolla, CA 92093, USA; \\ dtrinidad@ucsd.edu \\ 8 Institute for Mental Health Policy Research, Centre for Addiction and Mental Health, London, ON M5T 1R8, \\ Canada; Tara.EltonMarshall@camh.ca \\ 9 Dalla Lana School of Public Health, University of Toronto, Toronto, ON M5S 1A1, Canada \\ 10 Department of Epidemiology and Biostatistics, Schulich School of Medicine and Dentistry, \\ Western University, London, ON N6A 3K7, Canada \\ 11 Ontario Tobacco Research Unit, Toronto, ON M5S 2S1, Canada \\ 12 Department of Family Medicine and Community Health, Robert Wood Johnson Medical School, Rutgers, \\ Somerset, NJ 08873, USA; DanielA_Gundersen@dfci.harvard.edu \\ 13 Survey and Data Management Core, Dana-Farber Cancer Institute, Boston, MA 02215, USA \\ 14 School of Public Health and Health Systems, University of Waterloo, Waterloo, ON N2L 3G1, Canada; \\ geoffrey.fong@uwaterloo.ca \\ 15 Department of Psychology, University of Waterloo, Waterloo, ON N2L 3G1, Canada \\ 16 Ontario Institute for Cancer Research, Toronto, ON M5G 0A3, Canada \\ * Correspondence: karin.kasza@roswellpark.org; Tel.: +1-716-845-8085
}

Received: 1 October 2018; Accepted: 10 November 2018; Published: 14 November 2018

Abstract: More than half of adult tobacco users in the United States (U.S.) transitioned in tobacco product use between 2013-2014 and 2014-2015. We examine how characteristics of adult tobacco users in the U.S. relate to transitions in tobacco product use. Population Assessment of Tobacco and Health (PATH) Study data were analyzed from 12,862 adult current tobacco users who participated in Wave 1 (W1, 2013-2014) and Wave 2 (W2, 2014-2015). Three types of transitions were examined-(1) adding tobacco product(s); (2) switching to non-cigarette tobacco product(s); and (3) discontinuing all 
tobacco use-among those currently using: (1) any tobacco product; (2) cigarettes only (i.e., exclusive cigarette); and (3) cigarettes plus another tobacco product(s) (i.e., poly-cigarette). Multinomial logistic regression analyses determined relative risk of type of transition versus no transition as a function of demographic and tobacco use characteristics. Transitions in tobacco product use among adult tobacco users were common overall, but varied among different demographic groups, including by age, sex, sexual orientation, race/ethnicity, educational attainment, and poverty level. Further, cigarette smokers with higher dependence scores were more likely to add product(s) and less likely to discontinue tobacco use compared to those with low dependence scores. That high nicotine dependence is a barrier to discontinuing tobacco use adds evidence to support policy to lower nicotine content of cigarettes and to evaluate new products for their potential to reduce cigarette use.

Keywords: tobacco; transition; population; longitudinal; epidemiology; cigarettes; electronic nicotine delivery systems (ENDS); correlate; demographic; dependence

\section{Introduction}

As the tobacco product landscape in the United States (U.S.) expands [1-3] and prevalence of use of non-cigarette tobacco products increases [4-6], longitudinal data reveal that transitions in use of tobacco products are common among adult tobacco users in the U.S. That is, data from the Population Assessment of Tobacco and Health (PATH) Study indicate that over half of adult tobacco users transitioned in product use or combination of products used between 2013-2014 and 2014-2015, though cigarettes still remain the predominate type of tobacco product used by adults [7]. Understanding the population-level impact of the changing tobacco landscape requires determining both whether and how cigarette smokers and other tobacco product users transition in their product use over time, as well as whether transitions differ by demographic or tobacco use characteristics, such as frequency of use, number of products used, and indicators of nicotine dependence.

Prior research shows that age [7,8] and frequency of product use [9] are each associated with transitions in tobacco use, with younger adults and non-daily cigarette smokers being more likely to transition among tobacco products than their older adult and daily cigarette smoker counterparts. However, prior studies analyzing correlates of transitions among types of tobacco products have not considered the full range of new and emerging tobacco products available today, nor have they addressed a wider range of demographic characteristics that have historically been associated with tobacco use, such as race/ethnicity, sexual orientation, and socioeconomic status [10-15]. Additionally, a contemporary examination of how nicotine dependence relates to transitions in tobacco product use within the changing tobacco marketplace can help inform the U.S. Food and Drug Administration's plan to reduce the addictiveness of cigarettes by limiting their nicotine content [16].

This paper characterizes the demographic correlates (i.e., age, sex, sexual orientation, race/ethnicity, educational attainment, and poverty level) and tobacco use correlates (i.e., total number of products used, nicotine dependence, and type and frequency of product used) of transitions in tobacco product use in the U.S. over a one-year period (i.e., 2013-2014 to 2014-2015). Using Wave 1 (W1) and Wave 2 (W2) data from the PATH Study, we report relative likelihood of various types of transitions as a function of demographic and tobacco use correlates among all adult current tobacco users at W1, among adult current cigarette smokers who were not current users of other tobacco products at $\mathrm{W} 1$, and among adult current cigarette smokers who were also current users of at least one other type of tobacco product at W1. 


\section{Materials and Methods}

\subsection{Study Population}

The PATH Study is an ongoing, nationally-representative, longitudinal cohort study of tobacco use and health among adults and youths in the U.S. [17]. W1 data collection was conducted from 12 September 2013 through 14 December 2014 and W2 data collection was conducted from 23 October 2014 through 30 October 2015. The PATH Study recruitment employed a stratified address-based, area-probability sampling design at W1 that oversampled adult tobacco users, young adults (18 to 24 years), and African Americans. An in-person screener was used at W1 to select adults and youths from households for participation. At W1, the weighted response rate for the household screener was $54.0 \%$. Among households that were screened, the overall weighted response rate for the adult interview was $74.0 \%$ at W1 and $83.2 \%$ at W2. Audio computer-assisted self-interviews (ACASI) administered in English or Spanish were used to collect self-report information on tobacco-use patterns and associated health behaviors. All adult participants provided informed consent.

Data from 12,862 adult tobacco users (18+ years of age in W1) who participated in both W1 and W2 were used for this analysis. The weighted estimates presented here are representative of the non-institutionalized, civilian U.S. adult population through use of the probability sample and application of population and replicate weights that adjust for the complex study design characteristics (e.g., oversampling at W1) and nonresponse at W1 and W2. Nonresponse bias analyses for W1 and W2 are available at http:/ / doi.org/10.3886/ICPSR36231 [18]. Further details regarding the PATH Study design and methods are published elsewhere [17]. Details on survey interview procedures, questionnaires, sampling, weighting, and information on accessing the data are available at http: / / www.icpsr.umich.edu/icpsrweb/NAHDAP/series/00606 [19]. The study was conducted by Westat and approved by the Westat institutional review board (IRB Organization number IORG0000410, IRB number 00000695).

\subsection{Measures}

\subsubsection{Types of Tobacco Products}

Participants were provided with brief descriptions and pictures of 10 types of tobacco products (except cigarettes) and were asked about their use of the products at each wave, which were grouped for analysis into six types: cigarettes, ENDS (e-cigarettes at W1, and e-cigarettes, e-cigars, e-pipes, and e-hookah at W2), cigars (i.e., traditional cigars, cigarillos, filtered cigars), pipe tobacco, hookah, and smokeless tobacco (i.e., loose snus, moist snuff, dip, spit, chewing tobacco, snus pouches, dissolvable tobacco). At W2, non-cigarette tobacco product types were additionally grouped into combustible products (i.e., cigars, pipe tobacco, and hookah) and non-combustible products (i.e., ENDS and smokeless tobacco).

\subsubsection{Current Use of Tobacco Products}

At each wave, and for each type of tobacco product, participants were asked whether they now use the product every day, some days, or not at all. For hookah, participants were additionally asked whether they now use usually weekly or usually monthly. Current use at each wave was defined for cigarettes as having smoked at least 100 cigarettes in their lifetime and now smoking every day or somedays; for hookah as now smoking every day or somedays (including those who responded some days, usually weekly, or usually monthly; no threshold for units used); and for all other tobacco products as now smoking/using every day or somedays (no threshold for units used). For each tobacco product, a three-level current use variable was created to indicate daily use, someday use, or no use based on the criteria described above. The total number of products currently used was also calculated (complete data required). 


\subsubsection{Tobacco Product User Groups}

Tobacco product user groups were defined as follows: Any tobacco, defined as current use of any tobacco product(s); Non-cigarette tobacco, defined as current use of at least one tobacco product and no current use of cigarettes; Exclusive cigarettes, defined as current use of cigarettes and no current use of any other tobacco product; Poly-cigarettes, defined as current use of cigarettes and current use of at least one other tobacco product; Cigarettes plus ENDS, defined as current use of cigarettes and current use of ENDS (may also currently use any other tobacco product); Cigarettes plus combustible, defined as current use of cigarettes and current use of at least one other combustible tobacco product (may also currently use any non-combustible tobacco product); Cigarettes plus non-combustible, defined as current use of cigarettes and current use of at least one non-combustible tobacco product and no current use of any combustible tobacco product; No cigarettes and no ENDS, defined as no current use of cigarettes and no current use of ENDS (may currently use any other tobacco product); No tobacco, defined as no current use of any tobacco product. Some of these user groups overlap with each other to allow for the assessment of variations in user groups and transitions among them.

\subsubsection{Nicotine Dependence}

Sixteen items assessing symptoms of nicotine dependence were included in the PATH Study. Responses to these items were combined and scaled to produce an overall nicotine dependence score as described and validated by Strong and colleagues [20].

\subsubsection{Transitions in Tobacco Product Use}

Transitions in tobacco product use were determined among each of the following three tobacco user groups at W1 as defined above: any tobacco users, exclusive cigarette smokers, and poly-cigarette smokers. Among $\mathrm{W} 1$ any tobacco users $(\mathrm{N}=12,862)$, transitions in tobacco product use between waves were determined using three mutually exclusive transition groups: (1) no transition, defined as current use of the exact same tobacco product(s) at W1 and W2; (2) transition among tobacco product(s), defined as any difference in current use of tobacco product(s) at W1 and W2 (i.e., any transition in type of product(s) used, which could result in an increase, decrease, or no change in number of products used) except for the case of no current tobacco use at W2; and (3) discontinue tobacco use, defined as no current tobacco use at W2.

Among W1 exclusive cigarette smokers, transitions in tobacco product use between waves were determined with two sets of analyses: first, transitions were determined only with respect to ENDS using four mutually exclusive transition groups $(\mathrm{N}=4893)$ : (1) no transition, defined as cigarette use at W2 and no ENDS use at W2 (may use any other tobacco product at W2); (2) transition to cigarettes plus ENDS use; (3) switch to ENDS use, defined as ENDS use at W2 and no cigarette use at W2 (may use any other tobacco product at W2); and (4) transition to no cigarette use and no ENDS use at W2. Second, transitions in tobacco product use among exclusive cigarette smokers at W1 were determined with respect to all tobacco products using five mutually exclusive transition groups ( $\mathrm{N}=4846)$ : (1) no transition, defined as exclusive cigarette use at W2; (2) transition to cigarettes plus combustible use; (3) transition to cigarettes plus non-combustible use; (4) switch to non-cigarette tobacco use; and (5) discontinue tobacco use.

Among W1 poly-cigarette smokers $(\mathrm{N}=3946)$, transitions in tobacco product use between waves were determined using four mutually exclusive transition groups: (1) no transition, defined as poly-cigarette use at W2 (which may reflect the same or different combinations of products used at W2 as were used at W1); (2) transition to exclusive cigarette use; (3) transition to non-cigarette tobacco use; and (4) discontinue tobacco use. 


\subsubsection{Demographic Characteristics}

At W1, participants were categorized according to age group (i.e., 18-24, 25-39, 40-54, 55+), sex (male, female), sexual orientation (i.e., heterosexual/straight, gay/lesbian/bisexual/something else), race/ethnicity (i.e., non-Hispanic white, non-Hispanic black or African American, non-Hispanic other, Hispanic), educational attainment (i.e., less than high school or GED, high school graduate, some college (no degree) or Associates degree, Bachelor's degree or more), and household poverty level (i.e., below poverty level, at or near poverty level, at or above twice the poverty level, not reported). Missing data on race, ethnicity and education were imputed as described in the PATH Study Restricted Use Files User Guide (available at http:/ / www.icpsr.umich.edu/icpsrweb/NAHDAP/series/00606) [19].

\subsection{Statistical Analysis}

Rates of transitions in tobacco product use between waves were determined among (1) any tobacco users at W1; (2) exclusive cigarette smokers at W1; and (3) poly-cigarette smokers at W1. For each of these three user groups at W1, transition rates were also stratified by demographic characteristics (i.e., age group, sex, sexual orientation, race/ethnicity, educational attainment, and household poverty level), and tobacco use variables assessed at W1 (i.e., total number of products currently used (not relevant for exclusive cigarette smokers), nicotine dependence (stratified into quartiles), and type and frequency of products currently used (i.e., no use, someday use, daily use)). These stratification variables are hereafter referred to as "correlates".

Multinomial logistic regression analyses were used to determine the relative risk of being categorized into each transition group versus the "no transition" group as a function of correlates, using two models: Model 1 was adjusted for demographic variables only, and Model 2 was adjusted for all correlates (Model 1 consisted of separate analyses such that the analysis for each correlate was adjusted for demographic correlates only; Model 2 consisted of a single model that included all correlates at once). Analyses were conducted with Stata 14 software [21]. Estimates were weighted to represent the U.S. adult population and variances were estimated using the balanced repeated replication (BRR) method [22] with Fay's adjustment set to 0.3 to increase estimate stability [23]. The logit-transformation method was used to calculate standard errors for the proportions. Estimates based on a sample size of less than 50 or with a relative standard error of greater than $30 \%$ are flagged in the tables.

\section{Results}

\subsection{Any Tobacco User at W1}

Table 1 shows rates and relative likelihood of transitions in tobacco product use among those using any tobacco at W1, stratified by correlates, and relative risk ratios (RRR) indicate the relative probabilities of being in each transition group versus the "no transition" group as a function of correlates assessed at W1. Overall, $47.9 \%$ of tobacco users did not transition in use of tobacco product(s), 37.6\% transitioned among tobacco product(s), and $14.5 \%$ discontinued tobacco use. 
Table 1. Correlates of transitions in tobacco product use among 'any tobacco' users at Wave 1 (W1) of the Population Assessment of Tobacco and Health (PATH) Study $¥$

\begin{tabular}{|c|c|c|c|c|c|c|c|c|c|c|c|c|c|}
\hline & \multicolumn{13}{|c|}{ Transitions Assessed between W1 \& Wave 2 (W2) } \\
\hline & \multicolumn{3}{|c|}{$\begin{array}{l}\text { No transition (i.e.e, use the exact } \\
\text { same tobacco product(s) at W2 as } \\
\text { used at W1; referent group) }\end{array}$} & \multicolumn{5}{|c|}{$\begin{array}{l}\text { Transition among tobacco product(s) (i.e., any difference in use of tobacco } \\
\text { product(s) at W1 and W2 except for the case of no tobacco use at W2) }\end{array}$} & \multicolumn{5}{|c|}{ Discontinue tobacco use (i.e., no use of any tobacco product at W2 } \\
\hline & & & & & & & Model $1{ }^{1}$ & Model $2^{2}$ & & & & Model $1{ }^{1}$ & Model $2^{2}$ \\
\hline Correlates assessed at W1 & $\mathrm{N}$ & $\%$ & SE & $\mathrm{N}$ & $\%$ & $\mathrm{SE}$ & $\begin{array}{c}\text { RRR } \\
(95 \% \mathrm{CI})\end{array}$ & $\begin{array}{c}\text { RRR } \\
(95 \% \text { CI })\end{array}$ & $\mathrm{N}$ & $\%$ & SE & $\begin{array}{c}\text { RRR } \\
(95 \% \mathrm{CI})\end{array}$ & $\begin{array}{c}\text { RRR } \\
(95 \% \mathrm{CI})\end{array}$ \\
\hline Overall & 5947 & 47.9 & 0.55 & 5111 & 37.6 & 0.50 & & & 1804 & 14.5 & 0.37 & & \\
\hline \multicolumn{14}{|l|}{ Age group } \\
\hline $18-24$ & 942 & 27.0 & 0.84 & 1842 & 41.9 & 0.92 & ref & ref & 689 & 21.2 & 0.94 & ref & ref \\
\hline $25-39$ & 1745 & 43.9 & 0.89 & 1611 & 41.8 & 0.87 & $\begin{array}{c}0.5 \\
(0.5-0.6)\end{array}$ & $\begin{array}{c}0.7 \\
(0.6-0.8)\end{array}$ & 524 & 14.3 & 0.70 & $\begin{array}{c}0.4 \\
(0.3-0.5)\end{array}$ & $\begin{array}{c}0.9 \\
(0.7-1.1)\end{array}$ \\
\hline $40-54$ & 1776 & 55.3 & 0.98 & 1079 & 33.6 & 0.85 & $\begin{array}{c}0.3 \\
(0.3-0.4)\end{array}$ & $\begin{array}{c}0.5 \\
(0.5-0.6)\end{array}$ & 316 & 11.1 & 0.64 & $\begin{array}{c}0.3 \\
(0.2-0.3)\end{array}$ & $\begin{array}{c}0.7 \\
(0.6-0.9)\end{array}$ \\
\hline $55+$ & 1483 & 63.0 & 1.14 & 578 & 23.8 & 1.00 & $\begin{array}{c}0.2 \\
(0.2-0.2) \\
\end{array}$ & $\begin{array}{c}0.4 \\
(0.3-0.4)\end{array}$ & 275 & 13.3 & 0.86 & $(0.2-0.4)$ & $\begin{array}{c}0.8 \\
(0.6-1.0)\end{array}$ \\
\hline \multicolumn{14}{|l|}{ Sex } \\
\hline Male & 3177 & 45.8 & 0.74 & 3071 & 39.8 & 0.67 & ref & ref & 968 & 14.3 & 0.50 & ref & ref \\
\hline Female & 2768 & 51.0 & 0.87 & 2040 & 34.2 & 0.81 & $\begin{array}{c}0.7 \\
(0.7-0.8)\end{array}$ & $\begin{array}{c}0.9 \\
(0.8-1.1)\end{array}$ & 834 & 14.7 & 0.52 & $\begin{array}{c}0.9 \\
(0.8-1.1)\end{array}$ & $\begin{array}{c}1.3 \\
(1.1-1.5)\end{array}$ \\
\hline \multicolumn{14}{|l|}{ Sexual orientation } \\
\hline Heterosexual/Straight & 5500 & 48.7 & 0.57 & 4526 & 37.0 & 0.52 & ref & ref & 1614 & 14.3 & 0.38 & ref & ref \\
\hline Gay/lesbian/bisexual/something else & 361 & 36.2 & 1.75 & 519 & 47.7 & 1.73 & $\begin{array}{c}1.5 \\
(1.3-1.8)\end{array}$ & $\begin{array}{c}1.2 \\
(1.0-1.5)\end{array}$ & 157 & 16.1 & 1.42 & $\begin{array}{c}1.3 \\
(1.0-1.6)\end{array}$ & $\begin{array}{c}1.3 \\
(1.0-1.8)\end{array}$ \\
\hline \multicolumn{14}{|l|}{ Race/ethnicity } \\
\hline Non-Hispanic White & 4052 & 50.0 & 0.68 & 3226 & 37.1 & 0.61 & ref & ref & 1009 & 12.9 & 0.43 & ref & ref \\
\hline Non-Hispanic Black or African American & 839 & 48.7 & 1.51 & 692 & 37.0 & 1.37 & $\begin{array}{c}1.0 \\
(0.8-1.1) \\
\end{array}$ & $\begin{array}{c}0.9 \\
(0.8-1.1)\end{array}$ & 266 & 14.3 & 1.22 & $\begin{array}{c}1.3 \\
(1.0-1.6) \\
\end{array}$ & $\begin{array}{c}0.9 \\
(0.7-1.1)\end{array}$ \\
\hline Non-Hispanic other & 393 & 40.7 & 1.97 & 436 & 40.9 & 2.07 & $\begin{array}{c}1.1 \\
(0.9-1.4)\end{array}$ & $\begin{array}{c}0.9 \\
(0.7-1.2)\end{array}$ & 141 & 18.4 & 1.43 & $\begin{array}{c}1.5 \\
(1.2-1.8)\end{array}$ & $\begin{array}{c}1.2 \\
(0.9-1.5)\end{array}$ \\
\hline Hispanic & 663 & 38.6 & 1.17 & 757 & 39.4 & 1.45 & $\begin{array}{c}1.1 \\
(0.9-1.2)\end{array}$ & $\begin{array}{c}1.0 \\
(0.9-1.2)\end{array}$ & 388 & 22.0 & 1.32 & $\begin{array}{c}2.2 \\
(1.8-2.6)\end{array}$ & $\begin{array}{c}1.1 \\
(0.9-1.4)\end{array}$ \\
\hline \multicolumn{14}{|l|}{ Educational attainment } \\
\hline Less than high school or GED & 1490 & 49.7 & 1.13 & 1326 & 40.7 & 1.13 & ref & ref & 284 & 9.6 & 0.61 & ref & ref \\
\hline High school graduate & 1460 & 50.0 & 1.01 & 1282 & 37.6 & 1.03 & $\begin{array}{c}0.9 \\
(0.8-1.0)\end{array}$ & $\begin{array}{c}0.9 \\
(0.8-1.1)\end{array}$ & 395 & 12.5 & 0.59 & $\begin{array}{c}1.2 \\
(1.0-1.5)\end{array}$ & $\begin{array}{c}1.0 \\
(0.8-1.2)\end{array}$ \\
\hline Some college (no degree) or associates degree & 2126 & 45.6 & 0.86 & 1922 & 38.9 & 0.76 & $\begin{array}{c}1.0 \\
(0.9-1.1)\end{array}$ & $\begin{array}{c}0.9 \\
(0.8-1.0)\end{array}$ & 731 & 15.5 & 0.59 & $\begin{array}{c}1.6 \\
(1.3-2.0)\end{array}$ & $\begin{array}{c}1.1 \\
(0.8-1.3)\end{array}$ \\
\hline
\end{tabular}


Table 1. Cont.

\begin{tabular}{|c|c|c|c|c|c|c|c|c|c|c|c|c|c|}
\hline & \multicolumn{13}{|c|}{ Transitions Assessed between W1 \& Wave 2 (W2) } \\
\hline & \multicolumn{3}{|c|}{$\begin{array}{l}\text { No transition (i.e., use the exact } \\
\text { same tobacco product(s) at W2 as } \\
\text { used at } W 1 \text {; referent group) }\end{array}$} & \multicolumn{5}{|c|}{$\begin{array}{l}\text { Transition among tobacco product(s) (i.e., any difference in use of tobacco } \\
\text { product(s) at W1 and W2 except for the case of no tobacco use at W2) }\end{array}$} & \multicolumn{5}{|c|}{ Discontinue tobacco use (i.e., no use of any tobacco product at W2) } \\
\hline & & & & & & & Model $1{ }^{1}$ & Model $2^{2}$ & & & & Model $1^{1}$ & Model $2^{2}$ \\
\hline Bachelor's degree or more & 871 & 46.8 & 1.34 & 581 & 30.6 & 1.11 & $\begin{array}{c}0.8 \\
(0.7-1.0) \\
\end{array}$ & $\begin{array}{c}0.7 \\
(0.6-0.9) \\
\end{array}$ & 394 & 22.7 & 1.13 & $\begin{array}{c}2.5 \\
(2.0-3.2) \\
\end{array}$ & $\begin{array}{c}0.9 \\
(0.7-1.2) \\
\end{array}$ \\
\hline \multicolumn{14}{|l|}{ Poverty level } \\
\hline Below poverty level & 1919 & 43.8 & 0.98 & 2068 & 43.6 & 0.83 & ref & ref & 582 & 12.6 & 0.62 & ref & ref \\
\hline At or near poverty level & 1467 & 49.9 & 0.84 & 1159 & 37.3 & 0.86 & $\begin{array}{c}0.9 \\
(0.8-1.0)\end{array}$ & $\begin{array}{c}1.0 \\
(0.8-1.1)\end{array}$ & 377 & 12.9 & 0.67 & $\begin{array}{c}1.0 \\
(0.8-1.2)\end{array}$ & $\begin{array}{c}1.0 \\
(0.8-1.3)\end{array}$ \\
\hline At or above twice the poverty level & 2097 & 49.2 & 0.89 & 1511 & 33.8 & 0.80 & $\begin{array}{c}0.8 \\
(0.7-1.0) \\
\end{array}$ & $\begin{array}{c}0.9 \\
(0.8-1.1) \\
\end{array}$ & 701 & 17.0 & 0.66 & $\begin{array}{c}1.2 \\
(1.0-1.4) \\
\end{array}$ & $\begin{array}{c}1.0 \\
(0.8-1.3)\end{array}$ \\
\hline Not reported & 464 & 51.8 & 2.03 & 373 & 33.5 & 1.75 & $\begin{array}{c}0.7 \\
(0.6-0.9)\end{array}$ & $\begin{array}{c}0.9 \\
(0.7-1.1)\end{array}$ & 144 & 14.8 & 1.37 & $\begin{array}{c}1.0 \\
(0.8-1.4)\end{array}$ & $\begin{array}{c}1.0 \\
(0.7-1.5)\end{array}$ \\
\hline \multicolumn{14}{|l|}{ Total number of products currently used } \\
\hline 1 product & 4843 & 62.5 & 0.66 & 1708 & 20.2 & 0.53 & ref & ref & 1323 & 17.3 & 0.53 & ref & ref \\
\hline 2 or more products & 1104 & 24.5 & 0.78 & 3219 & 66.7 & 0.94 & $\begin{array}{c}7.4 \\
(6.6-8.3) \\
\end{array}$ & $\begin{array}{c}3.9 \\
(3.0-5.0)\end{array}$ & 418 & 8.8 & 0.55 & $\begin{array}{c}1.1 \\
(1.0-1.3)\end{array}$ & $\begin{array}{c}1.2 \\
(0.8-1.8)\end{array}$ \\
\hline \multicolumn{14}{|l|}{ Nicotine dependence ${ }^{3}$} \\
\hline 1st quartile & 1200 & 37.1 & 0.89 & 1101 & 29.5 & 0.80 & ref & ref & 1086 & 33.3 & 0.82 & ref & ref \\
\hline 2nd quartile & 1468 & 52.0 & 1.08 & 1205 & 37.9 & 1.05 & $\begin{array}{c}1.0 \\
(0.9-1.2)\end{array}$ & $\begin{array}{c}1.2 \\
(1.0-1.4)\end{array}$ & 308 & 10.1 & 0.59 & $\begin{array}{c}0.2 \\
(0.2-0.3)\end{array}$ & $\begin{array}{c}0.5 \\
(0.4-0.7)\end{array}$ \\
\hline 3rd quartile & 1771 & 53.4 & 1.07 & 1411 & 40.6 & 1.02 & $\begin{array}{c}1.2 \\
(1.0-1.3) \\
\end{array}$ & $\begin{array}{c}1.4 \\
(1.2-1.7) \\
\end{array}$ & 192 & 6.0 & 0.52 & $\begin{array}{c}0.2 \\
(0.1-0.2) \\
\end{array}$ & $\begin{array}{c}0.5 \\
(0.4-0.7) \\
\end{array}$ \\
\hline 4th quartile & 1411 & 51.4 & 1.10 & 1280 & 44.1 & 1.13 & $\begin{array}{c}1.4 \\
(1.3-1.7) \\
\end{array}$ & $\begin{array}{c}1.5 \\
(1.3-1.9) \\
\end{array}$ & 125 & 4.5 & 0.45 & $\begin{array}{c}0.1 \\
(0.1-0.2) \\
\end{array}$ & $\begin{array}{c}0.4 \\
(0.3-0.6)\end{array}$ \\
\hline \multicolumn{14}{|l|}{ Current use of cigarettes } \\
\hline No use & 1469 & 39.7 & 0.90 & 1390 & 31.6 & 0.76 & ref & ref & 1142 & 28.8 & 0.83 & ref & ref \\
\hline Someday use & 772 & 43.9 & 1.25 & 711 & 36.3 & 1.21 & $\begin{array}{c}1.1 \\
(0.9-1.2) \\
\end{array}$ & $\begin{array}{c}0.8 \\
(0.6-1.1) \\
\end{array}$ & 339 & 19.8 & 1.13 & $\begin{array}{c}0.6 \\
(0.5-0.7) \\
\end{array}$ & $\begin{array}{c}0.7 \\
(0.5-1.0) \\
\end{array}$ \\
\hline Daily use & 3706 & 53.7 & 0.74 & 3002 & 41.4 & 0.72 & $\begin{array}{c}1.2 \\
(1.0-1.3) \\
\end{array}$ & $\begin{array}{c}0.8 \\
(0.6-1.0)\end{array}$ & 322 & 4.9 & 0.30 & $\begin{array}{c}0.1 \\
(0.1-0.2)\end{array}$ & $\begin{array}{c}0.2 \\
(0.1-0.3)\end{array}$ \\
\hline \multicolumn{14}{|l|}{ Current use of ENDS } \\
\hline No use & 5156 & 53.1 & 0.62 & 3340 & 31.5 & 0.55 & ref & ref & 1486 & 15.4 & 0.43 & ref & ref \\
\hline Someday use & 535 & 24.1 & 1.00 & 1477 & 63.5 & 1.08 & $\begin{array}{c}4.4 \\
(3.8-5.0) \\
\end{array}$ & $\begin{array}{c}1.6 \\
(1.2-2.1) \\
\end{array}$ & 277 & 12.4 & 0.75 & $\begin{array}{c}1.7 \\
(1.4-2.0) \\
\end{array}$ & $\begin{array}{c}1.4 \\
(1.0-1.9)\end{array}$ \\
\hline Daily use & 256 & 46.1 & 2.39 & 287 & 47.6 & 2.27 & $\begin{array}{c}1.9 \\
(1.5-2.3)\end{array}$ & $\begin{array}{c}1.1 \\
(0.8-1.6)\end{array}$ & 36 & 6.3 & 1.09 & $\begin{array}{c}0.5 \\
(0.4-0.8)\end{array}$ & $\begin{array}{c}0.3 \\
(0.2-0.5)\end{array}$ \\
\hline
\end{tabular}


Table 1. Cont

\begin{tabular}{|c|c|c|c|c|c|c|c|c|c|c|c|c|c|}
\hline & \multicolumn{13}{|c|}{ Transitions Assessed between W1 \& Wave 2 (W2) } \\
\hline & \multicolumn{3}{|c|}{$\begin{array}{l}\text { No transition (i.e., use the exact } \\
\text { same tobacco product(s) at } W 2 \text { as } \\
\text { used at } W 1 \text {; referent group) }\end{array}$} & \multicolumn{5}{|c|}{$\begin{array}{l}\text { Transition among tobacco product(s) (i.e., any difference in use of tobacco } \\
\text { product(s) at W1 and W2 except for the case of no tobacco use at W2) }\end{array}$} & \multicolumn{5}{|c|}{ Discontinue tobacco use (i.e., no use of any tobacco product at $\mathrm{W} 2$ ) } \\
\hline & & & & & & & Model $1^{1}$ & Model $2^{2}$ & & & & Model $1^{1}$ & Model $2^{2}$ \\
\hline \multicolumn{14}{|c|}{$\begin{array}{l}\text { Current use of traditional } \\
\text { cigars/cigarillos/filtered cigars }\end{array}$} \\
\hline No use & 5033 & 57.5 & 0.62 & 2757 & 29.3 & 0.56 & ref & ref & 1166 & 13.2 & 0.42 & ref & ref \\
\hline Someday use & 772 & 25.8 & 0.94 & 1980 & 56.4 & 1.05 & $\begin{array}{c}4.2 \\
(3.8-4.8)\end{array}$ & $\begin{array}{c}2.0 \\
(1.6-2.5)\end{array}$ & 546 & 17.9 & 0.74 & $\begin{array}{c}2.8 \\
(2.4-3.2)\end{array}$ & $\begin{array}{c}1.4 \\
(1.1-1.9)\end{array}$ \\
\hline Daily use & 142 & 33.6 & 2.69 & 267 & 55.6 & 2.66 & $\begin{array}{c}3.7 \\
(2.8-4.9)\end{array}$ & $\begin{array}{c}1.6 \\
(1.2-2.2)\end{array}$ & 53 & 10.9 & 1.67 & $\begin{array}{c}1.7 \\
(1.1-2.5)\end{array}$ & $1.1(0.7-1.8)$ \\
\hline \multicolumn{14}{|c|}{ Current use of pipe } \\
\hline No use & 5864 & 49.3 & 0.57 & 4690 & 36.2 & 0.49 & ref & ref & 1740 & 14.5 & 0.40 & ref & ref \\
\hline Someday use & 65 & 14.6 & 1.86 & 385 & 70.6 & 2.50 & $\begin{array}{c}6.2 \\
(4.4-8.7)\end{array}$ & $\begin{array}{c}2.1 \\
(1.4-3.3)\end{array}$ & 60 & 14.8 & 2.00 & $\begin{array}{c}3.3 \\
(2.1-5.2)\end{array}$ & $\begin{array}{c}1.7 \\
(1.1-2.8)\end{array}$ \\
\hline Daily use & 18 & 40.7 & 7.14 & 28 & 55.3 & 7.98 & $\begin{array}{c}2.4 \\
(1.3-4.5)\end{array}$ & $\begin{array}{c}1.4 \\
(0.6-3.3)\end{array}$ & 2 & 4.0 & 2.76 & $\begin{array}{c}0.4 \\
(0.1-2.2)\end{array}$ & $\begin{array}{c}0.3 \\
(0.0-1.7)\end{array}$ \\
\hline \multicolumn{14}{|c|}{ Current use of hookah } \\
\hline No use & 5577 & 54.1 & 0.61 & 3649 & 33.5 & 0.52 & ref & ref & 1208 & 12.4 & 0.35 & ref & ref \\
\hline Someday use & 361 & 15.6 & 0.86 & 1430 & 59.1 & 1.31 & $\begin{array}{c}4.4 \\
(3.8-5.1)\end{array}$ & $\begin{array}{c}2.7 \\
(2.1-3.5)\end{array}$ & 585 & 25.4 & 1.07 & $\begin{array}{c}4.3 \\
(3.6-5.1)\end{array}$ & $\begin{array}{c}2.3 \\
(1.6-3.3)\end{array}$ \\
\hline Daily use & 9 & 21.9 & 6.80 & 29 & 62.2 & 7.79 & $\begin{array}{c}3.5 \\
(1.3-9.3)\end{array}$ & $\begin{array}{c}1.7 \\
(0.6-5.1)\end{array}$ & 7 & 15.9 & 6.30 & $\begin{array}{c}2.2 \\
(0.8-8.4) \\
\end{array}$ & $\begin{array}{c}1.1 \\
(0.2-5.6)\end{array}$ \\
\hline \multicolumn{14}{|c|}{$\begin{array}{l}\text { Current use of smokeless/snus } \\
\text { pouches/dissolvable }\end{array}$} \\
\hline No use & 5276 & 48.6 & 0.58 & 4267 & 36.3 & 0.54 & ref & ref & 1636 & 15.1 & 0.39 & ref & ref \\
\hline Someday use & 180 & 25.5 & 1.76 & 516 & 62.4 & 1.93 & $\begin{array}{c}2.8 \\
(2.3-3.4)\end{array}$ & $\begin{array}{c}1.6 \\
(1.3-2.0)\end{array}$ & 92 & 12.1 & 1.27 & $\begin{array}{c}1.4 \\
(1.1-1.9)\end{array}$ & $\begin{array}{c}1.1 \\
(0.8-1.6)\end{array}$ \\
\hline Daily use & 491 & 62.9 & 1.76 & 267 & 28.9 & 1.59 & $\begin{array}{c}0.6 \\
(0.5-0.7)\end{array}$ & $\begin{array}{c}0.6 \\
(0.5-0.7)\end{array}$ & 59 & 8.2 & 1.18 & $\begin{array}{c}0.5 \\
(0.3-0.6)\end{array}$ & $\begin{array}{c}0.4 \\
(0.2-0.5)\end{array}$ \\
\hline $\begin{array}{l}\text { \# Table } 1 \text { notes. Sample } \\
\text { Results from multinon } \\
\text { assessed at W1. Curre } \\
\text { everyday, somedays, o } \\
\text { estimate due to denom } \\
\text { adjusted for age, sex, } \\
\text { cumulative nicotine de }\end{array}$ & $\begin{array}{l}\text { aged } 1 \\
\text { ssion } \\
\text { ve def } \\
\text { l other } \\
\text { tive st } \\
\text { race } \\
\text { quarti }\end{array}$ & $\begin{array}{l}\text { ears } \\
\text { ses i } \\
\text { for ci } \\
\text { ducts } \\
\text { rrd of } \\
\text { icity, }\end{array}$ & $\begin{array}{l}\text { were' } \\
\text { ate the } \\
\text { ttes as } \\
\text { ow usi } \\
\text { estima } \\
\text { cation, } \\
\text { amon }\end{array}$ & $\begin{array}{l}\text { y toba } \\
\text { elative } \\
\text { aving } \\
\text { gever } \\
>30 \% \\
\text { overty } \\
\text { W1 ar }\end{array}$ & $\begin{array}{l}\text { users } \\
\text { obabil } \\
\text { ked a } \\
\text { y or s } \\
\text { Analys } \\
\text { el, tot } \\
\text { obaccs }\end{array}$ & $\begin{array}{l}11 \text { and } \\
\text { being } \\
\text { t } 100 \\
\text { lays. } \\
\text { justec } \\
\text { mber }\end{array}$ & $\begin{array}{l}\text { o participa } \\
\text { each } W 2 \mathrm{t} \\
\text { rettes in li } \\
\text { font indic } \\
\text { age, sex, } \\
\text { roducts us }\end{array}$ & $\begin{array}{l}\text { W2 }(\mathrm{N}=12 \\
\text { on group vs } \\
\text { and now sn } \\
\text { gnificant as } \\
\text { orientation, } \\
\text { otine deper }\end{array}$ & $\begin{array}{l}62) \text {; ar } \\
\text { he W2 } \\
\text { oking } \\
\text { ciatio } \\
\text { ace/et } \\
\text { lence, }\end{array}$ & $\begin{array}{l}\text { ses we } \\
\text { trans } \\
\text { yday } \\
\alpha=0 . \\
\text { city, ec } \\
\text { type }\end{array}$ & $\begin{array}{l}\text { ted us } \\
\text { n' gro } \\
\text { omed. } \\
\text { grey s. } \\
\text { ation, a } \\
\text { freque }\end{array}$ & $\begin{array}{l}\text { N2 longit } \\
\text { a functic } \\
\text { or hooka } \\
\text { d cells in } \\
\text { overty le } \\
\text { of produc }\end{array}$ & $\begin{array}{l}1 \text { weights. } \\
\text { correlates } \\
\text { ow using } \\
\text { unstable } \\
\text { Analyses } \\
\text { d. }{ }^{3} \text { Mean }\end{array}$ \\
\hline
\end{tabular}




\subsubsection{Correlates of Transitions among Tobacco Product(s)}

Young adults (i.e., those aged 18-24 years) were about five times more likely to transition among tobacco product(s) versus not transition than were adults aged 55+ years (Table 1, Model 1, $R R R=0.2,95 \% C I=0.2-0.2$ ), and were more than twice as likely to transition after adjustment for tobacco use correlates (Model 2). Those identifying as gay/lesbian/bisexual/something else were $20 \%$ more likely to transition among tobacco product(s) than those identifying as heterosexual/straight after adjustment for all correlates (Table 1, Model 2, RRR $=1.2,95 \% \mathrm{CI}=1.0-1.5$ ). Tobacco users with higher nicotine dependence at W1 were more likely to transition among tobacco product(s) versus not transition than were those with nicotine dependence in the first quartile at W1 after adjustment for all correlates (Table 1, Model 2). Poly-tobacco users at W1 were about four times more likely to transition among tobacco product(s) versus not transition than were exclusive tobacco users at W1 after adjustment for all correlates (Table 1, Model 2, RRR $=3.9,95 \% \mathrm{CI}=3.0-5.0$ ).

Someday users versus non-users were more likely to transition among tobacco product(s) across models for each type of tobacco product except cigarettes, and those who were daily users versus non-users of smokeless tobacco were less likely to transition among tobacco product(s) (Table 1 , Models 1-2).

\subsubsection{Correlates of Discontinuing Tobacco Use}

Young adults were more likely to discontinue tobacco use versus not transition than were older adults (Table 1, Model 1); however, the strength of this association was reduced/disappeared after adjustment for tobacco use correlates (Table 1, Model 2). Those identifying as gay/lesbian/bisexual/something else were 30\% more likely to discontinue tobacco use than those identifying as heterosexual/straight after adjustment for all correlates (Table 1, Model 2, RRR = 1.3, $95 \% \mathrm{CI}=1.0-1.8$ ). Tobacco users with higher nicotine dependence at $\mathrm{W} 1$ were less likely to discontinue tobacco use versus not transition than were those with nicotine dependence in the first quartile, and those who were daily users versus non-users of cigarettes, ENDS, or smokeless tobacco were also less likely to discontinue tobacco use, whereas those who were someday users versus non-users of cigars, pipe tobacco, or hookah were more likely to discontinue tobacco use regardless of adjustments (Table 1 , Models 1-2). Associations for other demographic and tobacco use correlates among any tobacco users are shown in Table 1.

\subsection{Exclusive Cigarette Smokers at W1}

Tables 2 and 3 show rates and relative likelihood of transitions in tobacco product use among exclusive cigarette smokers at W1, as a function of correlates assessed at W1, using two separate approaches to transitions: Table 2 shows transitions only with respect to ENDS and Table 3 shows transitions with respect to all tobacco products.

When considering transitions only with respect to ENDS (Table 2), the majority of exclusive cigarette smokers, $78.2 \%$, did not transition, $11.2 \%$ transitioned to cigarettes plus ENDS use, $1.5 \%$ switched to ENDS use, and 9.1\% transitioned to no cigarettes and no ENDS use. When considering transitions with respect to all tobacco products (Table 3), the majority of exclusive cigarette smokers, $72.8 \%$, did not transition, $6.3 \%$ transitioned to cigarettes plus combustible use, $10.3 \%$ transitioned to cigarettes plus non-combustible use, $1.9 \%$ switched to non-cigarette tobacco use, and $8.7 \%$ discontinued tobacco use. 
Table 2. Correlates of transitions in tobacco product use among 'exclusive cigarette' smokers at Wave 1 (W1); transitions defined only with respect to cigarettes and Electronic Nicotine Delivery Systems (ENDS) use t.

\begin{tabular}{|c|c|c|c|c|c|c|c|c|c|c|c|c|c|c|c|c|c|c|}
\hline & \multicolumn{18}{|c|}{ Transitions Assessed between W1 \& Wave 2 (W2) } \\
\hline & \multicolumn{3}{|c|}{$\begin{array}{c}\text { No transition a (i.e., cigarette use at } \\
\text { W2, no ENDS use at W2; referent } \\
\text { group) }\end{array}$} & \multicolumn{5}{|c|}{$\begin{array}{l}\text { Transition to cigarettes plus ENDS use } \\
\text { (i.e., cigarette use at W2 and ENDS use at W2) }\end{array}$} & \multicolumn{5}{|c|}{$\begin{array}{c}\text { Switch to ENDS use } \\
\text { (i.e., no cigarette use at W2, ENDS use at W2) }\end{array}$} & \multicolumn{5}{|c|}{$\begin{array}{l}\text { Transition to no cigarette and no ENDS use } \\
\text { (i.e., no cigarette use at W2, no ENDS use at W2) }\end{array}$} \\
\hline & & & & & & & Model 11 & Model 2 2 & & & & Model 11 & Model 2 2 & & & & Model $1{ }^{1}$ & Model 22 \\
\hline Correlates assessed at $\mathrm{W} 1$ & $\mathrm{~N}$ & $\%$ & $\mathrm{SE}$ & $\mathrm{N}$ & $\%$ & $\mathrm{SE}$ & $\begin{array}{c}\mathrm{RRR} \\
(95 \% \mathrm{CI}) \\
\end{array}$ & $\begin{array}{c}\mathrm{RRR} \\
(95 \% \mathrm{CI}) \\
\end{array}$ & $\mathrm{N}$ & $\%$ & SE & $\begin{array}{c}\mathrm{RRR} \\
(95 \% \mathrm{CI}) \\
\end{array}$ & $\begin{array}{c}\mathrm{RRR} \\
(95 \% \mathrm{CI}) \\
\end{array}$ & $\mathrm{N}$ & $\%$ & $\mathrm{SE}$ & $\begin{array}{c}\mathrm{RRR} \\
(95 \% \mathrm{CI}) \\
\end{array}$ & $\begin{array}{c}\mathrm{RRR} \\
(95 \% \mathrm{CI}) \\
\end{array}$ \\
\hline Overall & 3846 & 78.2 & 0.66 & 558 & 11.2 & 0.52 & & & 75 & 1.5 & 0.20 & & & 414 & 9.1 & 0.46 & & \\
\hline \multicolumn{19}{|l|}{ Age group } \\
\hline $18-24$ & 489 & 70.9 & 1.72 & 104 & 15.3 & 1.49 & $\begin{array}{l}\text { ref } \\
0.8\end{array}$ & $\begin{array}{l}\text { ref } \\
0.8\end{array}$ & 12 & 1.9 & 0.61 & $\begin{array}{l}\text { ref } \\
1.2\end{array}$ & $\begin{array}{ll}\text { ref } \\
1.1\end{array}$ & 74 & 12.0 & 1.20 & $\begin{array}{l}\text { ref } \\
0.7\end{array}$ & ref \\
\hline 40-54 & 1186 & $\begin{array}{ll}75.1 \\
79.9\end{array}$ & 1.23 & 189 & 12.0 & 0.98 & $\begin{array}{c}(0.6-1.1) \\
0.7 \\
(0.6-1.0)\end{array}$ & $\begin{array}{c}(0.6-1.0) \\
0.7 \\
(0.5-0.9)\end{array}$ & 14 & 1.0 & 0.28 & $\begin{array}{c}(0.5-2.7) \\
0.4 \\
(0.2-1.2)\end{array}$ & $\begin{array}{c}(0.5-2.5) \\
0.4 \\
(0.1-1.1)\end{array}$ & $\begin{array}{l}134 \\
99\end{array}$ & 7.1 & 0.86 & $\begin{array}{c}(0.5-0.9) \\
0.5 \\
(0.3-0.7)\end{array}$ & $\begin{array}{l}\frac{(0.6-1.1)}{0.8} \\
(0.5-1.1)\end{array}$ \\
\hline $55+$ & 1005 & 82.3 & 1.28 & 76 & 6.6 & 0.74 & $\begin{array}{c}0.4 \\
(0.3-0.6) \\
\end{array}$ & $\begin{array}{c}0.4 \\
(0.3-0.5) \\
\end{array}$ & 11 & 0.9 & 0.31 & $\begin{array}{c}0.4 \\
(0.2-1.1) \\
\end{array}$ & $\begin{array}{c}0.4 \\
(0.1-1.1) \\
\end{array}$ & 107 & 10.1 & 1.07 & $\begin{array}{c}0.7 \\
(0.5-1.0) \\
\end{array}$ & $\begin{array}{c}1.1 \\
(0.8-1.7) \\
\end{array}$ \\
\hline \multicolumn{19}{|l|}{ Sex } \\
\hline Male & 1708 & 79.3 & 0.90 & 207 & 9.7 & 0.70 & ref & ref & 40 & 1.8 & 0.30 & ref & ref & 182 & 9.2 & 0.72 & ref & ref \\
\hline Female & 2138 & 77.2 & 0.97 & 351 & 12.6 & 0.73 & $\begin{array}{c}1.3 \\
(1.1-1.6)\end{array}$ & $\begin{array}{c}1.3 \\
(1.0-1.6)\end{array}$ & 35 & 1.2 & 0.25 & $\begin{array}{c}0.6 \\
(0.4-1.1)\end{array}$ & $\begin{array}{c}0.6 \\
(0.4-1.0)\end{array}$ & 231 & 9.0 & 0.69 & $\begin{array}{c}1.1 \\
(0.8-1.4)\end{array}$ & $\begin{array}{c}1.1 \\
(0.8-1.5)\end{array}$ \\
\hline \multicolumn{19}{|l|}{ Sexual orientation } \\
\hline Heterosexual/Straight & 3544 & 78.5 & 0.69 & 495 & 10.8 & 0.52 & ref & ref & 72 & 1.6 & 0.22 & ref & ref & 377 & 9.1 & 0.48 & ref & ref \\
\hline Gay/lesbian/bisexual/something else & 239 & 71.9 & 3.05 & 58 & 18.0 & 2.57 & $\begin{array}{c}1.6 \\
(1.1-2.4) \\
\end{array}$ & $\begin{array}{c}1.6 \\
(1.1-2.3)\end{array}$ & 2 & 0.3 & 0.25 & $\begin{array}{c}0.2 \\
(0.0-1.3) \\
\end{array}$ & $\begin{array}{c}0.2 \\
(0.0-1.3) \\
(0)\end{array}$ & 30 & 9.9 & 2.13 & $\begin{array}{c}1.1 \\
(0.7-1.9)\end{array}$ & $\begin{array}{c}1.4 \\
(0.8-2.3) \\
\end{array}$ \\
\hline \multicolumn{19}{|l|}{ Race/ethnicity } \\
\hline Non-Hispanic White & 2519 & 77.2 & 0.77 & 421 & 12.3 & 0.63 & ref & ref & 60 & 1.8 & 0.29 & ref & ref & 258 & 8.6 & 0.53 & ref & ref \\
\hline $\begin{array}{l}\text { Non-Hispanic Black or African } \\
\text { American }\end{array}$ & 639 & 86.1 & 1.43 & 46 & 6.5 & 1.00 & $\begin{array}{c}0.5 \\
(0.3-0.7)\end{array}$ & $\begin{array}{c}0.5 \\
(0.4-0.8)\end{array}$ & 5 & 0.8 & 0.37 & $\begin{array}{c}0.5 \\
(0.1-1.5)\end{array}$ & $\begin{array}{c}0.5 \\
(0.1-1.5)\end{array}$ & 49 & 6.6 & 1.18 & $\begin{array}{c}0.8 \\
(0.6-1.2)\end{array}$ & $\begin{array}{c}0.6 \\
(0.4-0.9)\end{array}$ \\
\hline Non-Hispanic other & 233 & 69.8 & 3.37 & 37 & 13.3 & 2.50 & $\begin{array}{c}1.1 \\
(0.7-1.9)\end{array}$ & $\begin{array}{c}1.2 \\
(0.7-2.0)\end{array}$ & 4 & 0.6 & 0.32 & $\begin{array}{c}0.3 \\
(0.1-1.2)\end{array}$ & $\begin{array}{c}(0.1-1.3) \\
0.3 \\
(0.1-1.3)\end{array}$ & 35 & 16.3 & 2.63 & $\begin{array}{c}0.0-1.2) \\
(1.3-2.9)\end{array}$ & $\begin{array}{c}0.4-0.3) \\
1.5 \\
(1.0-2.3)\end{array}$ \\
\hline Hispanic & 455 & 78.8 & 1.85 & 54 & 8.3 & 1.24 & $\begin{array}{c}0.7 \\
(0.5-0.9)\end{array}$ & $\begin{array}{c}0.8 \\
(0.6-1.1)\end{array}$ & 6 & 1.0 & 0.35 & $\begin{array}{c}0.6 \\
(0.2-1.4)\end{array}$ & $\begin{array}{c}0.6 \\
(0.2-1.5)\end{array}$ & 72 & 11.9 & 1.54 & $\begin{array}{c}1.6 \\
(1.1-2.2)\end{array}$ & $\begin{array}{c}0.9 \\
(0.6-1.2)\end{array}$ \\
\hline \multicolumn{19}{|l|}{ Educational attainment } \\
\hline Less than high school or GED & 1160 & 81.4 & 1.06 & 157 & 10.9 & 0.91 & ref & ref & 13 & 0.8 & 0.26 & ref & ref & 86 & 6.9 & 0.82 & ref & ref \\
\hline High school graduate & 981 & 79.6 & 1.13 & 142 & 10.8 & 0.96 & $\begin{array}{c}0.9 \\
(0.7-1.2) \\
\end{array}$ & 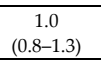 & 14 & 1.2 & 0.36 & $\begin{array}{c}1.4 \\
(0.6-3.4)\end{array}$ & $\begin{array}{c}1.4 \\
(0.6-3.5) \\
\end{array}$ & 101 & 8.3 & 0.71 & $\begin{array}{c}1.2 \\
(0.9-1.6)\end{array}$ & $\begin{array}{c}1.0 \\
(0.7-1.3) \\
\end{array}$ \\
\hline $\begin{array}{l}\text { Some college (no degree) or } \\
\text { associates degree }\end{array}$ & 1313 & 76.5 & 1.22 & 202 & 11.6 & 0.88 & $\begin{array}{c}0.9 \\
(0.7-1.3)\end{array}$ & $\begin{array}{c}1.0 \\
(0.7-1.3)\end{array}$ & 40 & 2.4 & 0.42 & $\begin{array}{c}2.5 \\
(1.1-5.7)\end{array}$ & $\begin{array}{c}2.5 \\
(1.1-5.7)\end{array}$ & 152 & 9.4 & 0.85 & $\begin{array}{c}1.3 \\
(0.9-1.8)\end{array}$ & $\begin{array}{c}1.0 \\
(0.7-1.4) \\
\end{array}$ \\
\hline Bachelor's degree or more & 392 & 71.8 & 2.19 & 57 & 11.3 & 1.34 & $\begin{array}{c}1.0 \\
(0.7-1.4) \\
\end{array}$ & $\begin{array}{c}1.1 \\
(0.8-1.6)\end{array}$ & 8 & 1.3 & 0.41 & $\begin{array}{c}1.5 \\
(0.5-4.0) \\
\end{array}$ & $\begin{array}{c}1.6 \\
(0.5-4.6) \\
\end{array}$ & 75 & 15.6 & 1.79 & $\begin{array}{c}2.0 \\
(1.3-2.9) \\
\end{array}$ & $\begin{array}{c}1.0 \\
(0.7-1.6) \\
(0.0)\end{array}$ \\
\hline \multicolumn{19}{|l|}{ Poverty level } \\
\hline Below poverty level & 1421 & 80.0 & 1.04 & 205 & 11.3 & 0.80 & ref & ref & 23 & 1.2 & 0.19 & ref & ref & 125 & 7.6 & 0.72 & ref & ref \\
\hline At or near poverty level & 1025 & 79.9 & 1.18 & 147 & 11.2 & 0.92 & $\begin{array}{c}1.0 \\
(0.8-1.4)\end{array}$ & $\begin{array}{c}1.0 \\
(0.8-1.4)\end{array}$ & 23 & 1.8 & 0.41 & $\begin{array}{c}1.3 \\
(0.7-2.2)\end{array}$ & $\begin{array}{c}1.2 \\
(0.7-2.1) \\
\end{array}$ & 93 & 7.1 & 0.81 & $\begin{array}{c}0.9 \\
(0.7-1.3)\end{array}$ & $\begin{array}{c}1.0 \\
(0.7-1.4) \\
\end{array}$ \\
\hline At or above twice the poverty level & 1091 & 74.7 & 1.37 & 165 & 11.4 & 1.01 & $\begin{array}{c}1.1 \\
(0.8-1.5)\end{array}$ & $\begin{array}{c}1.1 \\
(0.8-1.5)\end{array}$ & 26 & 1.9 & 0.41 & $\begin{array}{c}1.2 \\
(0.7-2.2)\end{array}$ & $\begin{array}{c}1.3 \\
(0.7-2.3)\end{array}$ & 160 & 12.1 & 0.97 & $\begin{array}{c}1.5 \\
(1.1-2.1)\end{array}$ & $\begin{array}{c}1.5 \\
(1.1-2.1)\end{array}$ \\
\hline Not reported & 309 & 79.6 & 2.46 & 41 & 10.1 & 1.76 & $\begin{array}{c}1.0 \\
(0.7-1.6)\end{array}$ & $\begin{array}{c}1.1 \\
(0.7-1.7)\end{array}$ & 3 & 0.6 & 0.36 & $\begin{array}{c}0.6 \\
(0.1-2.7)\end{array}$ & $\begin{array}{c}0.6 \\
(0.1-2.9)\end{array}$ & 36 & 9.7 & 1.77 & $\begin{array}{c}1.3 \\
(0.8-2.1)\end{array}$ & $\begin{array}{c}1.4 \\
(0.8-2.3)\end{array}$ \\
\hline \multicolumn{19}{|l|}{ Nicotine dependence $^{3}$} \\
\hline 1st quartile & 915 & 72.5 & 1.37 & 95 & 6.5 & 0.79 & ref & ref & 19 & 1.5 & 0.33 & ref & ref & 229 & 19.6 & 1.19 & ref & ref \\
\hline
\end{tabular}


Table 2. Cont.

\begin{tabular}{|c|c|c|c|c|c|c|c|c|c|c|c|c|c|c|c|c|c|c|}
\hline & \multicolumn{18}{|c|}{ Transitions Assessed between W1 \& Wave 2 (W2) } \\
\hline & \multirow{2}{*}{\multicolumn{3}{|c|}{$\begin{array}{c}\text { No transition a (i.e., cigarette use at } \\
\text { W2, no ENDS use at W2; referent } \\
\text { group) }\end{array}$}} & \multicolumn{5}{|c|}{$\begin{array}{l}\text { Transition to cigarettes plus ENDS use } \\
\text { (i.e., cigarette use at W2 and ENDS use at W2) }\end{array}$} & \multicolumn{5}{|c|}{$\begin{array}{c}\text { Switch to ENDS use } \\
\text { (i.e., no cigarette use at W2, ENDS use at W2) }\end{array}$} & \multicolumn{5}{|c|}{$\begin{array}{l}\text { Transition to no cigarette and no ENDS use } \\
\text { (i.e., no cigarette use at W2, no ENDS use at W2) }\end{array}$} \\
\hline & & & & & & & Model 11 & Model 22 & & & & Model 11 & Model 22 & & & & Model 11 & Model 22 \\
\hline 2nd quartile & 932 & 80.4 & 1.31 & 128 & 11.2 & 0.98 & $\begin{array}{c}1.6 \\
(1.1-2.2) \\
\end{array}$ & $\begin{array}{c}1.4 \\
(0.9-2.0) \\
\end{array}$ & 15 & 1.3 & 0.35 & $\begin{array}{c}0.9 \\
(0.4-2.0)\end{array}$ & $\begin{array}{c}0.8 \\
(0.3-1.8)\end{array}$ & 79 & 7.2 & 0.97 & $\begin{array}{c}0.3 \\
(0.2-0.5)\end{array}$ & $\begin{array}{c}0.5 \\
(0.4-0.8)\end{array}$ \\
\hline 3rd quartile & 993 & 78.7 & 1.21 & 184 & 14.8 & 1.02 & $\begin{array}{c}2.1 \\
(1.5-2.9)\end{array}$ & $\begin{array}{c}1.8 \\
(1.2-2.5) \\
\end{array}$ & 24 & 2.0 & 0.48 & $\begin{array}{c}1.4 \\
(0.7-3.0) \\
\end{array}$ & $\begin{array}{c}1.2 \\
(0.5-2.7) \\
\end{array}$ & 51 & 4.5 & 0.64 & $\begin{array}{c}0.2 \\
(0.2-0.3) \\
\end{array}$ & $\begin{array}{c}0.4 \\
(0.3-0.7) \\
\end{array}$ \\
\hline 4th quartile & 951 & 81.6 & 1.24 & 149 & 12.8 & 1.08 & $\begin{array}{c}1.8 \\
(1.2-2.5) \\
\end{array}$ & $\begin{array}{c}1.5 \\
(1.0-2.2) \\
\end{array}$ & 16 & 1.4 & 0.39 & $\begin{array}{c}1.0 \\
(0.4-2.5) \\
\end{array}$ & $\begin{array}{c}0.9 \\
(0.3-2.3) \\
\end{array}$ & 48 & 4.2 & 0.72 & $\begin{array}{c}0.2 \\
(0.1-0.3) \\
\end{array}$ & $\begin{array}{c}0.4 \\
(0.3-0.6) \\
\end{array}$ \\
\hline \multicolumn{19}{|l|}{ Current use of $\mathrm{c}$} \\
\hline Someday use & 627 & 67.9 & 1.77 & 60 & 6.0 & 0.85 & ref & ref & 14 & 1.3 & 0.30 & ref & ref & 209 & 24.8 & 1.62 & ref & ref \\
\hline Daily use & 3219 & 80.6 & 0.68 & 498 & 12.4 & 0.59 & $\begin{array}{c}1.9 \\
(1.3-2.7)\end{array}$ & $\begin{array}{c}1.5 \\
(1.0-2.2)\end{array}$ & 61 & 1.6 & 0.25 & $\begin{array}{c}1.4 \\
(0.7-2.6)\end{array}$ & $\begin{array}{c}1.4 \\
(0.7-2.7)\end{array}$ & 205 & 5.5 & 0.43 & $\begin{array}{c}0.2 \\
(0.1-0.2)\end{array}$ & $\begin{array}{c}0.3 \\
(0.2-0.4)\end{array}$ \\
\hline
\end{tabular}

1 Table 2 notes. Sample includes adults aged 18+ years who were 'exclusive cigarette' smokers at W1 and also participated in W2 ( $\mathrm{N}=4893$ ); analyses weighted using W2 longitudinal weights. Results from multinomial logistic regression analyses indicate the relative probability of being in each W2 transition group vs the W2 'no transition' group as a function of correlates assessed at W1. Current use at each wave defined for cigarettes as having smoked at least 100 cigarettes in lifetime and now smoking everyday or somedays; for hookah as now using everyday, somedays, or monthly; for all other products as now using everyday or somedays. 'Exclusive cigarette' use defined as current use of cigarettes and no current use of any other tobacco product. Bold font indicates significant association at $\alpha=0.05$; grey shaded cells indicate unstable estimate due to denominator $<50$ or relative standard of the estimate $>30 \%$. ${ }^{1}$ Analyses adjusted for age, sex, sexual orientation, race/ethnicity, education, and poverty level. ${ }^{2}$ Analyses adjusted for age, sex, sexual orientation, race/ethnicity, education, poverty level, tobacco dependence, and frequency of cigarette use. ${ }^{3}$ Mean cumulative tobacco dependence score; quartiles determined among W1 'exclusive cigarette' smokers. ${ }^{a}$ No transition is defined only with respect to cigarettes and ENDS use; therefore, no transition may include adding combustible or smokeless tobacco products(s) at W2. 
Table 3. Correlates of transitions in tobacco product use among 'exclusive cigarette' smokers at Wave 1 (W1); transitions defined with respect to all tobacco products 1.

\begin{tabular}{|c|c|c|c|c|c|c|c|c|c|c|c|c|c|c|c|c|c|c|c|c|c|c|c|}
\hline & \multicolumn{23}{|c|}{ Transitions Assessed between W1 \& Wave 2 (W2) } \\
\hline & \multirow{2}{*}{\multicolumn{3}{|c|}{$\begin{array}{l}\text { No transition a } \\
\text { (i.e., exclusive cigarette use } \\
\text { at W2; referent group) }\end{array}$}} & \multicolumn{5}{|c|}{$\begin{array}{l}\text { Transition to cigarettes plus combustible use } \\
\text { (i.e., cigarette use and use of at least one other } \\
\text { combustible tobacco product at W2; may also use } \\
\text { any non-combustible tobacco product(s) at W2) }\end{array}$} & \multicolumn{5}{|c|}{$\begin{array}{l}\text { Transition to cigarettes plus non-combustible } \\
\text { use only (i.e., cigarette use and use of at least one } \\
\text { non-combustible tobacco product at W2; does } \\
\text { not use any combustible tobacco product at W2) }\end{array}$} & \multicolumn{5}{|c|}{$\begin{array}{l}\text { Switch to non-cigarette tobacco use } \\
\text { (i.e., no cigarette use at } \mathrm{W} 2 \text {, but use of any } \\
\text { other tobacco product at } \mathrm{W} 2 \text { ) }\end{array}$} & \multicolumn{5}{|c|}{$\begin{array}{c}\text { Discontinue tobacco use } \\
\text { (i.e., no use of any tobacco product at W2) }\end{array}$} \\
\hline & & & & & & & Model 11 & Model 22 & & & & Model 11 & Model 22 & & & & Model 11 & Model 2 2 & & & & Model 1 1 & Model $2^{2}$ \\
\hline Correlates assessed at $\mathrm{W} 1$ & $\mathrm{~N}$ & $\%$ & SE & $\mathrm{N}$ & $\%$ & SE & $\begin{array}{c}\mathrm{RRR} \\
(95 \% \mathrm{CI})\end{array}$ & $\begin{array}{c}\mathrm{RRR} \\
(95 \% \mathrm{CI})\end{array}$ & $\mathrm{N}$ & $\%$ & SE & $\begin{array}{c}\mathrm{RRR} \\
(95 \% \mathrm{CI})\end{array}$ & $\begin{array}{c}\text { RRR } \\
(95 \% \mathrm{CI})\end{array}$ & $\mathrm{N}$ & $\%$ & SE & $\begin{array}{c}\text { RRR } \\
(95 \% \mathrm{CI})\end{array}$ & $\begin{array}{c}\mathrm{RRR} \\
(95 \% \mathrm{CI})\end{array}$ & $\mathrm{N}$ & $\%$ & SE & $\begin{array}{c}\text { RRR } \\
(95 \% \mathrm{CI})\end{array}$ & $\begin{array}{c}\text { RRR } \\
(95 \% \mathrm{CI})\end{array}$ \\
\hline \multirow{2}{*}{\multicolumn{24}{|c|}{$\begin{array}{l}\text { Overall } \\
\text { Age group }\end{array}$}} \\
\hline & & & & & & & & & & & & & & & & & & & & & & & \\
\hline $18-24$ & 404 & 60.8 & 2.01 & 91 & 13.2 & 1.31 & ref & ref & 77 & 11.9 & 1.37 & ref & ref & 17 & 2.7 & 0.73 & ref & ref & 67 & 11.4 & 1.31 & ref & ref \\
\hline 25-39 & 1064 & 69.5 & 1.31 & 107 & 6.7 & 0.68 & $\begin{array}{c}0.5 \\
(0.3-0.6)\end{array}$ & $\begin{array}{c}0.4 \\
(0.3-0.6)\end{array}$ & 170 & 12.0 & 1.05 & $\begin{array}{c}0.9 \\
(0.7-1.3)\end{array}$ & $\begin{array}{c}0.9 \\
(0.6-1.2)\end{array}$ & 43 & 2.9 & 0.53 & $\begin{array}{c}0.9 \\
(0.4-1.9)\end{array}$ & $\begin{array}{c}0.9 \\
(0.4-1.8)\end{array}$ & 127 & 8.9 & 0.93 & $\begin{array}{c}0.6 \\
(0.4-0.8)\end{array}$ & $\begin{array}{c}0.8 \\
(0.5-1.1)\end{array}$ \\
\hline $40-54$ & 1109 & 74.7 & 1.25 & 88 & 5.9 & 0.71 & $\begin{array}{c}0.4 \\
(0.3-0.6) \\
\end{array}$ & $\begin{array}{c}0.4 \\
(0.3-0.6) \\
\end{array}$ & 174 & 11.3 & 1.03 & $\begin{array}{c}0.8 \\
(0.6-1.1) \\
\end{array}$ & $\begin{array}{c}0.7 \\
(0.5-1.0) \\
\end{array}$ & 16 & 1.2 & 0.27 & $\begin{array}{c}0.3 \\
(0.1-0.8) \\
\end{array}$ & $\begin{array}{c}0.3 \\
(0.1-0.7)\end{array}$ & 97 & 7.0 & 0.84 & $\begin{array}{c}0.5 \\
(0.3-0.7)\end{array}$ & $\begin{array}{c}0.7 \\
(0.5-1.1) \\
\end{array}$ \\
\hline $55+$ & 947 & 78.1 & 1.27 & 55 & 4.2 & 0.68 & $\begin{array}{c}0.3 \\
(0.2-0.4) \\
\end{array}$ & $\begin{array}{c}0.3 \\
(0.2-0.4) \\
\end{array}$ & 75 & 6.7 & 0.72 & $\begin{array}{c}0.5 \\
(0.3-0.7) \\
\end{array}$ & $\begin{array}{c}0.4 \\
(0.3-0.6) \\
\end{array}$ & 16 & 1.4 & 0.34 & $\begin{array}{c}0.4 \\
(0.2-0.8) \\
\end{array}$ & $\begin{array}{c}0.4 \\
(0.2-0.8) \\
\end{array}$ & 102 & 9.6 & 1.05 & $\begin{array}{c}0.6 \\
(0.4-1.0) \\
\end{array}$ & $\begin{array}{c}1.1 \\
(0.7-1.6) \\
\end{array}$ \\
\hline \multicolumn{24}{|l|}{ Sex } \\
\hline Male & 1513 & 71.6 & 0.99 & 192 & 8.2 & 0.64 & ref & ref & 189 & 9.2 & 0.72 & ref & ref & 49 & 2.3 & 0.32 & ref & ref & 172 & 8.8 & 0.69 & ref & ref \\
\hline Female & 2011 & 73.9 & 0.99 & 149 & 4.6 & 0.37 & $\begin{array}{c}0.5 \\
(0.4-0.7)\end{array}$ & $\begin{array}{c}0.5 \\
(0.4-0.6)\end{array}$ & 307 & 11.4 & 0.74 & $\begin{array}{c}1.2 \\
(0.9-1.4)\end{array}$ & $\begin{array}{c}1.1 \\
(0.9-1.4)\end{array}$ & 43 & 1.6 & 0.30 & $\begin{array}{c}0.7 \\
(0.4-1.1)\end{array}$ & $\begin{array}{c}0.6 \\
(0.4-1.1)\end{array}$ & 220 & 8.6 & 0.67 & $\begin{array}{c}1.0 \\
(0.8-1.3)\end{array}$ & $\begin{array}{c}1.1 \\
(0.8-1.4)\end{array}$ \\
\hline \multicolumn{24}{|l|}{ Sexual orientation } \\
\hline $\begin{array}{l}\text { Heterosexual/ } \\
\text { Straight }\end{array}$ & 3262 & 73.2 & 0.73 & 299 & 6.0 & 0.39 & ref & ref & 443 & 10.1 & 0.58 & ref & ref & 88 & 2.0 & 0.22 & ref & ref & 358 & 8.7 & 0.47 & ref & ref \\
\hline $\begin{array}{l}\text { Gay/lesbian/bisexual/somethi } \\
\text { ng else }\end{array}$ & 205 & 63.2 & 3.17 & 36 & 11.1 & 2.07 & $\begin{array}{c}2.0 \\
(1.2-3.2) \\
\end{array}$ & $\begin{array}{c}2.0 \\
(1.2-3.3) \\
\end{array}$ & 49 & 15.8 & 2.52 & $\begin{array}{c}1.7 \\
(1.1-2.5) \\
\end{array}$ & $\begin{array}{c}1.6 \\
(1.1-2.5) \\
\end{array}$ & 3 & 0.6 & 0.34 & $\begin{array}{c}0.3 \\
(0.1-1.2) \\
\end{array}$ & $\begin{array}{c}0.3 \\
(0.1-1.3) \\
\end{array}$ & 28 & 9.4 & 2.09 & $\begin{array}{c}1.2 \\
(0.7-2.0) \\
\end{array}$ & $\begin{array}{c}1.5 \\
(0.9-2.5) \\
\end{array}$ \\
\hline \multicolumn{24}{|l|}{ Race/ethnicity } \\
\hline Non-Hispanic White & 2332 & 72.2 & 0.78 & 198 & 5.7 & 0.45 & ref & ref & 387 & 11.8 & 0.67 & ref & ref & 68 & 2.1 & 0.28 & ref & ref & 246 & 8.3 & 0.53 & ref & ref \\
\hline $\begin{array}{l}\text { Non-Hispanic Black or African } \\
\text { American }\end{array}$ & 563 & 78.2 & 1.81 & 77 & 9.3 & 1.06 & $\begin{array}{c}1.3 \\
(1.0-1.7) \\
\end{array}$ & $\begin{array}{c}1.4 \\
(1.0-1.9)\end{array}$ & 33 & 4.9 & 0.84 & $\begin{array}{c}0.4 \\
(0.3-0.6)\end{array}$ & $\begin{array}{c}0.4 \\
(0.3-0.7)\end{array}$ & 11 & 1.6 & 0.53 & $\begin{array}{c}0.8 \\
(0.4-1.9)\end{array}$ & $\begin{array}{c}0.9 \\
(0.4-2.0)\end{array}$ & 44 & 6.0 & 1.10 & $\begin{array}{c}0.8 \\
(0.5-1.2)\end{array}$ & $\begin{array}{c}0.6 \\
(0.4-0.9)\end{array}$ \\
\hline Non-Hispanic other & 209 & 64.8 & 3.53 & 27 & 7.2 & 1.60 & $\begin{array}{c}1.2 \\
(0.7-2.1)\end{array}$ & $\begin{array}{c}1.2 \\
(0.7-2.1)\end{array}$ & 30 & 10.9 & 2.36 & $\begin{array}{c}1.0 \\
(0.6-1.7)\end{array}$ & $\begin{array}{c}1.1 \\
(0.6-1.8)\end{array}$ & 6 & 2.0 & 1.29 & $\begin{array}{c}0.9 \\
(0.2-3.9)\end{array}$ & $\begin{array}{c}0.9 \\
(0.2-3.8)\end{array}$ & 33 & 15.1 & 2.66 & $\begin{array}{c}1.9 \\
(1.2-2.9)\end{array}$ & $\begin{array}{c}1.5 \\
(0.9-2.3)\end{array}$ \\
\hline Hispanic & 420 & 73.7 & 2.11 & 39 & 6.0 & 1.15 & $\begin{array}{c}0.8 \\
(0.5-1.4) \\
\end{array}$ & $\begin{array}{c}0.9 \\
(0.5-1.5) \\
\end{array}$ & 46 & 7.5 & 1.18 & $\begin{array}{c}0.6 \\
(0.4-2.9) \\
\end{array}$ & $\begin{array}{c}0.7 \\
(0.5-1.1) \\
\end{array}$ & 7 & 1.1 & 0.38 & $\begin{array}{c}0.6 \\
(0.2-1.3) \\
\end{array}$ & $\begin{array}{c}0.6 \\
(0.3-1.3) \\
\end{array}$ & 70 & 11.7 & 1.55 & $\begin{array}{c}1.6 \\
(1.1-2.2) \\
\end{array}$ & $\begin{array}{c}0.9 \\
(0.6-1.3) \\
\end{array}$ \\
\hline \multicolumn{24}{|l|}{ Educational attainment } \\
\hline Less than high school or GED & 1042 & 74.1 & 1.12 & 116 & 7.8 & 0.74 & ref & ref & 145 & 10.3 & 0.82 & ref & ref & 16 & 1.1 & 0.28 & ref & ref & 83 & 6.7 & 0.79 & ref & ref \\
\hline High school graduate & 907 & 74.6 & 1.34 & 80 & 6.0 & 0.81 & $\begin{array}{c}0.8 \\
(0.6-1.2)\end{array}$ & $\begin{array}{c}0.9 \\
(0.6-1.3)\end{array}$ & 123 & 10.1 & 1.00 & $\begin{array}{c}0.9 \\
(0.7-1.1)\end{array}$ & $\begin{array}{c}0.9 \\
(0.7-1.2)\end{array}$ & 19 & 1.7 & 0.40 & $\begin{array}{c}1.4 \\
(0.7-2.9)\end{array}$ & $\begin{array}{c}1.4 \\
(0.7-3.0)\end{array}$ & 93 & 7.7 & 0.74 & $\begin{array}{c}1.1 \\
(0.8-1.5)\end{array}$ & $\begin{array}{c}0.9 \\
(0.6-1.2)\end{array}$ \\
\hline $\begin{array}{l}\text { Some college (no degree) or } \\
\text { associates degree }\end{array}$ & 1208 & 71.9 & 1.29 & 112 & 5.6 & 0.64 & $\begin{array}{c}0.8 \\
(0.6-1.1)\end{array}$ & $\begin{array}{c}0.8 \\
(0.6-1.2)\end{array}$ & 178 & 10.6 & 0.94 & $\begin{array}{c}0.9 \\
(0.7-1.2)\end{array}$ & $\begin{array}{c}0.9 \\
(0.7-1.2) \\
\end{array}$ & 45 & 2.7 & 0.43 & $\begin{array}{c}2.1 \\
(1.1-4.3)\end{array}$ & $\begin{array}{c}2.1 \\
(1.0-4.2)\end{array}$ & 146 & 9.2 & 0.87 & $\begin{array}{c}1.2 \\
(0.9-1.8) \\
\end{array}$ & $\begin{array}{c}0.9 \\
(0.6-1.3) \\
\end{array}$ \\
\hline Bachelor's degree or more & 367 & 67.2 & 2.20 & 33 & 6.0 & 1.18 & $\begin{array}{c}1.1 \\
(0.7-1.7)\end{array}$ & $\begin{array}{c}1.1 \\
(0.7-1.9)\end{array}$ & 50 & 9.9 & 1.36 & $\begin{array}{c}0.8 \\
(0.6-1.3)\end{array}$ & $\begin{array}{c}1.0 \\
(0.6-1.5) \\
\end{array}$ & 12 & 2.3 & 0.76 & $\begin{array}{c}2.0 \\
(0.9-4.5)\end{array}$ & $\begin{array}{c}2.0 \\
(0.9-4.7)\end{array}$ & 71 & 14.5 & 1.72 & $\begin{array}{c}1.8 \\
(1.2-2.8)\end{array}$ & $\begin{array}{c}0.9 \\
(0.6-1.5) \\
\end{array}$ \\
\hline \multicolumn{24}{|l|}{$\begin{array}{l}\text { Poverty level } \\
\end{array}$} \\
\hline Below poverty level & 1276 & 72.9 & 1.29 & 164 & 8.6 & 0.77 & ref & & 169 & 9.6 & 0.76 & & & 28 & 1.5 & 0.25 & ref & & 120 & 7.4 & 0.72 & ref & \\
\hline At or near poverty level & 942 & 74.7 & 1.26 & 76 & 5.7 & 0.70 & $\begin{array}{c}0.7 \\
(0.5-1.0)\end{array}$ & $\begin{array}{c}0.7 \\
(0.5-1.1)\end{array}$ & 137 & 10.8 & 0.97 & $\begin{array}{c}1.2 \\
(0.9-1.5)\end{array}$ & $\begin{array}{c}1.2 \\
(0.9-1.5)\end{array}$ & 28 & 2.2 & 0.42 & $\begin{array}{c}1.3 \\
(0.8-2.3)\end{array}$ & $\begin{array}{c}1.3 \\
(0.8-2.2)\end{array}$ & 85 & 6.6 & 0.76 & $\begin{array}{c}0.9 \\
(0.6-1.2)\end{array}$ & $\begin{array}{c}0.9 \\
(0.6-1.3)\end{array}$ \\
\hline $\begin{array}{c}\text { At or above twice the poverty } \\
\text { level }\end{array}$ & 1022 & 70.4 & 1.35 & 76 & 4.9 & 0.64 & $\begin{array}{c}0.7 \\
(0.4-1.0)\end{array}$ & $\begin{array}{c}0.7 \\
(0.4-1.0)\end{array}$ & 152 & 10.8 & 1.00 & $\begin{array}{c}1.2 \\
(0.9-1.6)\end{array}$ & $\begin{array}{c}1.2 \\
(0.9-1.6)\end{array}$ & 30 & 2.2 & 0.46 & $\begin{array}{c}1.2 \\
(0.6-2.2)\end{array}$ & $\begin{array}{c}1.2 \\
(0.7-2.3)\end{array}$ & 156 & 11.8 & 0.98 & $\begin{array}{c}1.5 \\
(1.1-2.1)\end{array}$ & $\begin{array}{c}1.5 \\
(1.1-2.1) \\
\end{array}$ \\
\hline Not reported & 284 & 75.2 & 2.78 & 25 & 5.2 & 1.08 & $\begin{array}{c}0.7 \\
(0.4-1.1) \\
\end{array}$ & $\begin{array}{c}0.7 \\
(0.4-1.1) \\
\end{array}$ & 38 & 9.7 & 1.71 & $\begin{array}{c}1.1 \\
(0.7-1.7) \\
\end{array}$ & $\begin{array}{c}1.2 \\
(0.8-1.9) \\
\end{array}$ & 6 & 1.4 & 0.56 & $\begin{array}{c}1.1 \\
(0.4-2.9) \\
\end{array}$ & $\begin{array}{c}1.2 \\
(0.4-3.1) \\
\end{array}$ & 32 & 8.5 & 1.71 & $\begin{array}{c}1.1 \\
(0.6-1.9) \\
\end{array}$ & $\begin{array}{c}1.2 \\
(0.7-2.1) \\
\end{array}$ \\
\hline \multicolumn{24}{|l|}{ Nicotine dependence $^{3}$} \\
\hline 1st quartile & 842 & \begin{tabular}{ll|}
68.1 \\
\end{tabular} & $\begin{array}{l}1.46 \\
\end{array}$ & 72 & 4.8 & 0.65 & ref & ref & 85 & 6.0 & 0.76 & ref & ref & 25 & 1.8 & 0.38 & ref & ref & 221 & 19.3 & 1.21 & ref & ref \\
\hline
\end{tabular}


Table 3. Cont.

\begin{tabular}{|c|c|c|c|c|c|c|c|c|c|c|c|c|c|c|c|c|c|c|c|c|c|c|c|}
\hline & \multicolumn{23}{|c|}{ Transitions Assessed between W1 \& Wave 2 (W2) } \\
\hline & \multirow{2}{*}{\multicolumn{3}{|c|}{$\begin{array}{l}\text { No transition a } \\
\text { (i.e., exclusive cigarette use } \\
\text { at W2; referent group) }\end{array}$}} & \multicolumn{5}{|c|}{$\begin{array}{l}\text { Transition to cigarettes plus combustible use } \\
\text { (i.e., cigarette use and use of at least one other } \\
\text { combustible tobacco product at } \mathrm{W} 2 \text {; may also use } \\
\text { any non-combustible tobacco product(s) at W2) }\end{array}$} & \multicolumn{5}{|c|}{$\begin{array}{l}\text { Transition to cigarettes plus non-combustible } \\
\text { use only (i.e., cigarette use and use of at least one } \\
\text { non-combustible tobacco product at } \mathrm{W} 2 \text {; does } \\
\text { not use any combustible tobacco product at } \mathrm{W} 2 \text { ) }\end{array}$} & \multicolumn{5}{|c|}{$\begin{array}{l}\text { Switch to non-cigarette tobacco use } \\
\text { (i.e., no cigarette use at } \mathrm{W} 2 \text {, but use of any } \\
\text { other tobacco product at } \mathrm{W} 2 \text { ) }\end{array}$} & \multicolumn{5}{|c|}{$\begin{array}{l}\text { Discontinue tobacco use } \\
\text { (i.e., no use of any tobacco product at W2) }\end{array}$} \\
\hline & & & & & & & Model 11 & Model 22 & & & & Model 11 & Model $2^{2}$ & & & & Model 11 & Model $2^{2}$ & & & & Model $1{ }^{1}$ & Model 22 \\
\hline 2nd quartile & 860 & 75.1 & 1.46 & 78 & 6.4 & 0.76 & $\begin{array}{c}1.3 \\
(0.8-1.9)\end{array}$ & $\begin{array}{c}1.4 \\
(0.9-2.1)\end{array}$ & 112 & 10.1 & 0.96 & $\begin{array}{c}1.5 \\
(1.0-2.1)\end{array}$ & $\begin{array}{c}1.3 \\
(0.9-1.8) \\
\end{array}$ & 20 & 1.9 & 0.46 & $\begin{array}{c}1.1 \\
(0.5-2.3)\end{array}$ & $\begin{array}{c}1.1 \\
(0.4-2.8)\end{array}$ & 73 & 6.6 & 0.87 & $\begin{array}{c}0.3 \\
(0.2-0.4) \\
\end{array}$ & $\begin{array}{c}0.5 \\
(0.3-0.7) \\
\end{array}$ \\
\hline 3rd quartile & 904 & 72.6 & 1.30 & 94 & 7.1 & 0.70 & $\begin{array}{c}1.5 \\
(1.1-2.1)\end{array}$ & $\begin{array}{c}1.7 \\
(1.1-2.5)\end{array}$ & 168 & 14.0 & 1.04 & $\begin{array}{c}2.1 \\
(1.5-3.0)\end{array}$ & $\begin{array}{c}1.7 \\
(1.2-2.5) \\
\end{array}$ & 26 & 2.2 & 0.50 & $\begin{array}{c}1.4 \\
(0.7-2.7)\end{array}$ & $\begin{array}{c}1.5 \\
(0.7-3.2)\end{array}$ & 47 & 4.1 & 0.67 & $\begin{array}{c}0.2 \\
(0.1-0.3)\end{array}$ & $\begin{array}{c}0.4 \\
(0.3-0.6)\end{array}$ \\
\hline 4th quartile & 871 & 75.5 & 1.37 & 88 & 6.9 & 0.73 & $\begin{array}{c}1.5 \\
(1.0-2.3) \\
\end{array}$ & $\begin{array}{c}1.7 \\
(1.0-2.6) \\
\end{array}$ & 130 & 11.9 & 1.22 & $\begin{array}{c}1.7 \\
(1.2-2.5) \\
\end{array}$ & $\begin{array}{c}1.4 \\
(0.9-2.1)\end{array}$ & 20 & 1.8 & 0.43 & $\begin{array}{c}1.2 \\
(0.6-2.5) \\
\end{array}$ & $\begin{array}{c}1.3 \\
(0.5-3.3)\end{array}$ & 45 & 3.9 & 0.64 & $\begin{array}{c}0.2 \\
(0.1-0.3) \\
\end{array}$ & $\begin{array}{c}0.4 \\
(0.2-0.6) \\
\end{array}$ \\
\hline \multicolumn{24}{|l|}{ Current use of cigaret } \\
\hline Someday use & 569 & 63.2 & 1.75 & 58 & 5.7 & 0.83 & ref & ref & 49 & 5.0 & 0.76 & ref & ref & 19 & 2.1 & 0.45 & ref & ref & 202 & 24.1 & 1.69 & ref & ref \\
\hline Daily use & 2955 & 75.0 & 0.73 & 283 & 6.5 & 0.44 & $\begin{array}{c}1.1 \\
(0.7-1.5)\end{array}$ & $\begin{array}{c}0.8 \\
(0.5-1.3)\end{array}$ & 447 & 11.5 & 0.64 & $\begin{array}{c}2.1 \\
(1.4-3.0)\end{array}$ & $\begin{array}{c}1.7 \\
(1.1-2.5)\end{array}$ & 73 & 1.9 & 0.24 & $\begin{array}{c}1.0 \\
(0.6-1.8)\end{array}$ & $\begin{array}{c}0.9 \\
(0.4-1.8)\end{array}$ & 191 & 5.1 & 0.42 & $\begin{array}{c}0.2 \\
(0.1-0.2)\end{array}$ & $\begin{array}{c}0.3 \\
(0.2-0.4)\end{array}$ \\
\hline
\end{tabular}

¥ Table 3 notes. Sample includes adults aged 18+ years who were 'exclusive cigarette' smokers at W1 and also participated in W2 ( $\mathrm{N}=4893$ ); analyses weighted using W2 longitudinal weights. Results from multinomial logistic regression analyses indicate the relative probability of being in each W2 transition group vs the W2 'no transition' group as a function of correlates assessed at W1. Current use at each wave defined for cigarettes as having smoked at least 100 cigarettes in lifetime and now smoking everyday or somedays; for hookah as now using everyday, somedays, or monthly; for all other products as now using everyday or somedays. 'Exclusive cigarette' use defined as current use of cigarettes and no current use of any other tobacco product. Bold font indicates significant association at $\alpha=0.05$; grey shaded cells indicate unstable estimate due to denominator $<50$ or relative standard of the estimate $>30 \%$. ${ }^{1}$ Analyses adjusted for age, sex, sexual orientation, race/ethnicity, education, and poverty level. ${ }^{2}$ Analyses adjusted for age, sex, sexual orientation, race/ethnicity, education, poverty level, tobacco dependence, and frequency of cigarette use. ${ }^{3}$ Mean cumulative tobacco dependence score; quartiles determined among W1 'exclusive cigarette' smokers. ${ }^{a}$ No transition is defined only with respect to cigarettes and ENDS use; therefore, no transition may include adding combustible or smokeless tobacco products(s) at W2. 


\subsubsection{Correlates of Transitions to Cigarettes Plus ENDS Use (Table 2)}

Among exclusive cigarette smokers, young adults were more than twice as likely to transition to cigarettes plus ENDS use versus not transition compared to those aged 55+ years (Table 2, Model 2, $\mathrm{RRR}=0.4,95 \% \mathrm{CI}=0.3-0.5)$, and about 1.4 times more likely to transition compared to those aged 40-54 years (Model 2, RRR $=0.7,95 \% \mathrm{CI}=0.5-0.9$ ), female smokers were more likely than male smokers (Model 2, RRR $=1.395 \% \mathrm{CI}=1.0-1.6$ ), smokers who identified as gay $/$ lesbian $/ \mathrm{bisexual} /$ something else were more likely than those who identified as heterosexual/straight (Model 2, $\mathrm{RRR}=1.6,95 \% \mathrm{CI}=1.1-2.3$ ), and African American smokers were half as likely as non-Hispanic white smokers (Model 2, RRR $=0.5,95 \% \mathrm{CI}=0.4-0.8$ ), to transition to cigarettes plus ENDS use versus not transition regardless of adjustments (Table 2). Those in the third quartile of nicotine dependence at W1 were more likely than those in the first quartile to transition to cigarettes plus ENDS use regardless of adjustments (Table 2, Model 2, RRR $=1.8,95 \% \mathrm{CI}=1.2-2.5$ ), and daily cigarette smokers were more likely than someday cigarette smokers to transition to cigarettes plus ENDS use regardless of adjustments (Model 2, RRR $=1.5,95 \% \mathrm{CI}=1.0-2.2$ ).

\subsubsection{Correlates of Transitions to Cigarettes Plus Combustible Use (Table 3)}

Among exclusive cigarette smokers, young adults were about 2-3 times more likely to transition to cigarettes plus combustible use versus not transition compared to their older adult counterparts regardless of adjustments (Table 3, Model 2 estimates ranging from $\mathrm{RRR}=0.4,95 \% \mathrm{CI}=0.3-0.6$ for those aged 25-39 years and for those aged $40-54$ years, to $R R R=0.3,95 \% \mathrm{CI}=0.2-0.4$ for those aged 55+ years), female smokers were about half as likely as male smokers (Model 2, RRR $=0.5$, $95 \% \mathrm{CI}=0.4-0.6$ ), and smokers who identified as gay/lesbian/bisexual/something else were about twice as likely as those who identified as heterosexual/straight (Model 2, RRR $=2.0,95 \% \mathrm{CI}=1.2-3.3$ ) to transition to cigarettes plus combustible use regardless of adjustments. Those in the third or fourth quartiles of nicotine dependence at W1 were more likely than those in the first quartile to transition to cigarettes plus combustible use regardless of adjustments (Table 3, Model 2, RRR $=1.7,95 \% \mathrm{CI}=1.1-2.5$ for third quartile; $R R R=1.7,95 \% \mathrm{CI}=1.0-2.6$ for fourth quartile). There was no difference in likelihood of transitioning to cigarettes plus combustible use between daily versus someday cigarette smokers at W1.

\subsubsection{Tobacco Product Switching (Tables 2 and 3)}

Among exclusive cigarette smokers, 1.5\% switched to ENDS (alone or in combination with another non-cigarette tobacco product, Table 2) and 1.9\% switched to any non-cigarette tobacco product use (which could include ENDS, Table 3). Most stratified estimates for switching transitions in Tables 2 and 3 were based on a sample size of less than 50 or had a relative standard error of greater than $30 \%$ and are therefore unstable.

3.2.4. Correlates of Transitions to No Cigarettes and No ENDS Use (Table 2) and to No Tobacco Use (Table 3)

Among exclusive cigarette smokers, African American smokers were less likely than non-Hispanic white smokers to transition to no cigarettes and no ENDS use (Table 2, Model 2, RRR $=0.6$, $95 \% \mathrm{CI}=0.4-0.9$ ), and to discontinue all tobacco use (Table 3, Model 2, RRR $=0.6,95 \% \mathrm{CI}=0.4-0.9$ ) when adjusting for all correlates. Those at or above twice the poverty level were $50 \%$ more likely than those below the poverty level to transition to no cigarettes and no ENDS use (Table 2, Model 2, $\mathrm{RRR}=1.5,95 \% \mathrm{CI}=1.1-2.1$ ) and to discontinue all tobacco use (Table 3, Model 2, RRR = 1.5, $95 \% \mathrm{CI}=1.1-2.1)$ regardless of adjustments. Associations varied across adjustments for other demographic characteristics.

Exclusive cigarette smokers with higher nicotine dependence were far less likely than were those with nicotine dependence in the first quartile, and daily cigarette smokers were also far less likely than 
someday cigarette smokers, to transition to no cigarettes and no ENDS use (Table 2), and to discontinue all tobacco use (Table 3) regardless of adjustments.

\subsection{Poly-Cigarette Smokers at W1}

Table 4 shows rates and relative likelihood of transitions in tobacco product use among poly-cigarette smokers at $\mathrm{W} 1$, as a function of correlates assessed at $\mathrm{W} 1$. Overall, $54.5 \%$ of poly-cigarette smokers did not transition in use of tobacco products, $32.5 \%$ transitioned to exclusive cigarette use, $6.7 \%$ transitioned to non-cigarette tobacco use, and $6.3 \%$ discontinued tobacco use

\subsubsection{Correlates of Transition to Exclusive Cigarette Use}

Among poly-cigarette smokers, young adults were about half as likely to transition to exclusive cigarette use versus not transition compared to those aged 40-54 years regardless of adjustments (Table 4, Model 2, RRR $=1.5,95 \% \mathrm{CI}=1.1-1.9$ ), female smokers were more likely than male smokers (Model 2, RRR $=1.4,95 \% \mathrm{CI}=1.1-1.6)$, smokers with a bachelor's degree or more were less likely than those with less than high school/GED education (Model 2, RRR $=0.6,95 \% \mathrm{CI}=0.5-0.9$ ), and those at or above twice the poverty level were more likely than those below the poverty level (Model 2, $R R R=1.5,95 \% C I=1.2-1.8$ ), to transition to exclusive cigarette use regardless of adjustments.

Those who were using three or more products at W1 were less likely than those using two products (Model 2, RRR $=0.6,95 \% \mathrm{CI}=0.4-0.8$ ), daily ENDS users were less likely than ENDS nonusers, daily cigar users were less likely than cigar non-users (Model 2, RRR $=0.3,95 \% \mathrm{CI}=0.2-0.5$ for ENDS use and for cigar use), and someday smokeless users were less likely than smokeless nonusers (Model 2, $\mathrm{RRR}=0.6,95 \% \mathrm{CI}=0.4-0.8)$ to transition to exclusive cigarette use versus not transition regardless of adjustments.

\subsubsection{Correlates of Transition to Non-Cigarette Tobacco Use}

Among poly-cigarette smokers, those at or above the poverty level were more likely to transition to non-cigarette tobacco use compared to those below the poverty level regardless of adjustments (Model 2, RRR $=1.5,95 \% \mathrm{CI}=1.0-2.3$ for those at or near the poverty level; $\mathrm{RRR}=2.0,95 \% \mathrm{CI}=1.3-3.1$ for those at or above twice the poverty level), and daily cigarette smokers were less likely than someday cigarette smokers to transition to non-cigarette tobacco use regardless of adjustments (Model $2, \mathrm{RRR}=0.4,95 \% \mathrm{CI}=0.2-0.5$ ). Associations varied across adjustments for other correlates (Table 4 ).

\subsubsection{Correlates of Discontinuing Tobacco Use}

Among poly-cigarette smokers, female smokers were more likely than male smokers (Table 4 , Model 2, RRR $=1.6,95 \% \mathrm{CI}=1.1-2.3)$, Hispanic smokers were more likely than non-Hispanic white smokers (Model 2, $\mathrm{RRR}=1.7,95 \% \mathrm{CI}=1.0-2.7)$, and daily cigarette smokers were less likely than someday cigarette smokers (Model $2, \mathrm{RRR}=0.3,95 \% \mathrm{CI}=0.2-0.5$ ), to discontinue tobacco use versus not transition regardless of adjustments. Associations for other correlates varied across adjustments as shown in Table 4, including for number of products used at W1 and nicotine dependence at W1, both of which were negatively related to discontinuing tobacco use when adjusting only for demographic characteristics, but did not have robust and significant associations with discontinuing tobacco use after adjusting for all tobacco use variables. 
Table 4. Correlates of transitions in tobacco product use among 'poly cigarette' smokers at Wave 1 (W1) 1.

\begin{tabular}{|c|c|c|c|c|c|c|c|c|c|c|c|c|c|c|c|c|c|c|}
\hline & \multicolumn{18}{|c|}{ Transitions assessed between Wave $1 \&$ Wave 2 (W2) } \\
\hline & \multicolumn{3}{|c|}{$\begin{array}{l}\text { No transition a } \\
\text { (i.e., poly cigarette use } \\
\text { at W2; referent group) }\end{array}$} & \multicolumn{5}{|c|}{$\begin{array}{l}\text { Transition to exclusive cigarette } \\
\text { (i.e., cigarette use at W2, no use of any other } \\
\text { tobacco product at W2) }\end{array}$} & \multicolumn{5}{|c|}{$\begin{array}{l}\text { Transition to non-cigarette tobacco use } \\
\text { (i.e., no cigarette use at } \mathrm{W} 2, \text { but use of any other } \\
\text { tobacco product(s) at W2; } 56.4 \% \text { of people in this } \\
\text { transition group were ENDS users at } \mathrm{W} 2 \text { ) }\end{array}$} & \multicolumn{5}{|c|}{$\begin{array}{l}\text { Discontinue tobacco use } \\
\text { (i.e., no use of any tobacco product at W2) }\end{array}$} \\
\hline & & & & & & & Model $1^{1}$ & Model $2^{2}$ & & & & Model $1^{1}$ & Model $2^{2}$ & & & & Model $1^{1}$ & Model $2^{2}$ \\
\hline Correlates assessed at W1 & $\mathrm{N}$ & $\%$ & SE & $\mathrm{N}$ & $\%$ & SE & $\begin{array}{c}\mathrm{RRR} \\
(95 \% \mathrm{CI}) \\
\end{array}$ & $\begin{array}{c}\mathrm{RRR} \\
(95 \% \mathrm{CI})\end{array}$ & $\mathrm{N}$ & $\%$ & SE & $\begin{array}{c}\mathrm{RRR} \\
(95 \% \mathrm{CI})\end{array}$ & $\begin{array}{c}\mathrm{RRR} \\
(95 \% \mathrm{CI}) \\
\end{array}$ & $\mathrm{N}$ & $\%$ & SE & $\begin{array}{c}\mathrm{RRR} \\
(95 \% \mathrm{CI})\end{array}$ & $\begin{array}{c}\mathrm{RRR} \\
(95 \% \mathrm{CI}) \\
\end{array}$ \\
\hline Overall & 2195 & 54.5 & 0.92 & 1248 & 32.5 & 0.88 & & & 261 & 6.7 & 0.49 & & & 242 & 6.3 & 0.46 & & \\
\hline \multicolumn{19}{|l|}{ Age group } \\
\hline $18-24$ & 719 & 60.2 & 1.45 & 292 & 23.5 & 1.35 & ref & ref & 93 & 8.0 & 0.80 & ref & ref & 88 & 8.4 & 1.02 & ref & ref \\
\hline $25-39$ & 740 & 55.7 & 1.52 & 427 & 31.6 & 1.40 & $\begin{array}{c}1.4 \\
(1.1-1.7) \\
\end{array}$ & $\begin{array}{c}1.2 \\
(1.0-1.6)\end{array}$ & 85 & 6.6 & 0.87 & $\begin{array}{c}0.8 \\
(0.5-1.1)\end{array}$ & $\begin{array}{c}1.0 \\
(0.6-1.5)\end{array}$ & 84 & 6.0 & 0.67 & $\begin{array}{c}0.8 \\
(0.5-1.1)\end{array}$ & $\begin{array}{c}1.0 \\
(0.7-1.4) \\
\end{array}$ \\
\hline $40-54$ & 479 & 50.4 & 1.78 & 346 & 39.0 & 1.66 & $\begin{array}{c}1.8 \\
(1.4-2.2) \\
\end{array}$ & $\begin{array}{c}1.5 \\
(1.1-1.9) \\
\end{array}$ & 49 & 5.7 & 0.91 & $\begin{array}{c}0.7 \\
(0.5-1.1)\end{array}$ & $\begin{array}{c}0.9 \\
(0.5-1.4) \\
\end{array}$ & 41 & 4.9 & 0.75 & $\begin{array}{c}0.7 \\
(0.4-1.1) \\
\end{array}$ & $\begin{array}{c}0.8 \\
(0.5-1.4) \\
\end{array}$ \\
\hline $55+$ & 256 & 50.2 & 2.50 & 182 & 36.4 & 2.31 & $\begin{array}{c}1.7 \\
(1.3-2.3)\end{array}$ & $\begin{array}{c}1.3 \\
(0.9-1.9)\end{array}$ & 34 & 6.9 & 1.24 & $\begin{array}{c}0.9 \\
(0.6-1.4)\end{array}$ & $\begin{array}{c}1.1 \\
(0.7-1.8)\end{array}$ & 29 & 6.6 & 1.38 & $\begin{array}{c}0.9 \\
(0.5-1.7)\end{array}$ & $\begin{array}{c}1.2 \\
(0.6-2.4)\end{array}$ \\
\hline \multicolumn{19}{|l|}{ Sex } \\
\hline Male & 1405 & 58.1 & 1.19 & 629 & 28.5 & 1.02 & ref & ref & 191 & 7.9 & 0.62 & ref & ref & 123 & 5.5 & 0.58 & ref & ref \\
\hline Female & 790 & 48.2 & 1.47 & 619 & 39.4 & 1.54 & $\begin{array}{c}1.8 \\
(1.5-2.1) \\
\end{array}$ & $\begin{array}{c}1.4 \\
(1.1-1.6)\end{array}$ & 70 & 4.6 & 0.67 & $\begin{array}{c}0.8 \\
(0.5-1.1)\end{array}$ & $\begin{array}{c}0.8 \\
(0.6-1.2) \\
\end{array}$ & 119 & 7.7 & 0.78 & $\begin{array}{c}1.7 \\
(1.2-2.3) \\
\end{array}$ & $\begin{array}{c}1.6 \\
(1.1-2.3) \\
\end{array}$ \\
\hline \multicolumn{19}{|l|}{ Sexual orientation } \\
\hline Heterosexual/Straight & 1938 & 54.6 & 1.00 & 1106 & 32.5 & 0.89 & ref & ref & 235 & 6.9 & 0.52 & ref & ref & 202 & 6.0 & 0.48 & ref & ref \\
\hline $\begin{array}{l}\text { Gay/lesbian/bisexual/ } \\
\text { something else }\end{array}$ & 244 & 57.8 & 2.58 & 117 & 29.7 & 2.64 & $\begin{array}{c}0.8 \\
(0.6-1.0)\end{array}$ & $\begin{array}{c}0.9 \\
(0.6-1.2)\end{array}$ & 23 & 4.8 & 1.26 & $\begin{array}{c}0.7 \\
(0.4-1.3)\end{array}$ & $\begin{array}{c}0.7 \\
(0.4-1.4)\end{array}$ & 33 & 7.7 & 1.36 & $\begin{array}{c}1.0 \\
(0.6-1.6)\end{array}$ & $\begin{array}{c}1.1 \\
(0.6-1.7)\end{array}$ \\
\hline \multicolumn{19}{|l|}{ Race/ethnicity } \\
\hline Non-Hispanic White & 1485 & 55.3 & 1.12 & 813 & 32.2 & 1.07 & ref & ref & 180 & 7.1 & 0.67 & ref & ref & 137 & 5.4 & 0.51 & ref & ref \\
\hline $\begin{array}{l}\text { Non-Hispanic Black or } \\
\text { African American }\end{array}$ & 253 & 53.5 & 2.67 & 168 & 36.4 & 2.45 & $\begin{array}{c}1.2 \\
(0.9-1.6)\end{array}$ & $\begin{array}{c}1.1 \\
(0.8-1.4)\end{array}$ & 24 & 5.0 & 1.20 & $\begin{array}{c}0.9 \\
(0.5-1.6)\end{array}$ & $\begin{array}{c}0.7 \\
(0.4-1.4) \\
\end{array}$ & 27 & 5.1 & 1.12 & $\begin{array}{c}1.1 \\
(0.7-1.9)\end{array}$ & $\begin{array}{c}0.8 \\
(0.4-1.4) \\
\end{array}$ \\
\hline Non-Hispanic other & 202 & 57.0 & 3.66 & 99 & 28.4 & 3.46 & $\begin{array}{c}1.0 \\
(0.7-1.4)\end{array}$ & $\begin{array}{c}0.8 \\
(0.6-1.3)\end{array}$ & 17 & 4.4 & 1.12 & $\begin{array}{c}0.6 \\
(0.3-1.1)\end{array}$ & $\begin{array}{c}0.5 \\
(0.3-1.0) \\
\end{array}$ & 25 & 10.2 & 2.30 & $\begin{array}{c}2.0 \\
(1.1-3.4) \\
\end{array}$ & $\begin{array}{c}1.7 \\
(0.9-3.0) \\
\end{array}$ \\
\hline Hispanic & 255 & 48.9 & 2.50 & 168 & 32.9 & 2.32 & $\begin{array}{c}1.2 \\
(1.0-1.6) \\
\end{array}$ & $\begin{array}{c}1.1 \\
(0.8-1.4) \\
\end{array}$ & 40 & 7.4 & 1.12 & $\begin{array}{c}1.4 \\
(0.9-2.1) \\
\end{array}$ & $\begin{array}{c}1.1 \\
(0.7-1.9) \\
\end{array}$ & 53 & 10.8 & 1.55 & $\begin{array}{c}2.6 \\
(1.7-4.0) \\
\end{array}$ & $\begin{array}{c}1.7 \\
(1.0-2.7) \\
\end{array}$ \\
\hline \multicolumn{19}{|l|}{ Educational attainment } \\
\hline Less than high school or GED & 591 & 54.9 & 1.46 & 361 & $\begin{array}{llll}35.7 & & \end{array}$ & 1.50 & ref & ref & 48 & 4.6 & 0.84 & ref & ref & 43 & 4.8 & $\begin{array}{ll}0.82 \\
\end{array}$ & ref & ref \\
\hline High school graduate & 549 & 54.8 & 1.96 & 299 & 33.2 & 1.95 & $\begin{array}{c}0.9 \\
(0.7-1.2)\end{array}$ & $\begin{array}{c}0.9 \\
(0.7-1.1)\end{array}$ & 67 & 7.2 & 1.06 & $\begin{array}{c}1.5 \\
(0.9-2.6)\end{array}$ & $\begin{array}{c}1.4 \\
(0.8-2.5)\end{array}$ & 48 & 4.7 & 0.75 & $\begin{array}{c}1.0 \\
(0.6-1.6)\end{array}$ & $\begin{array}{c}0.8 \\
(0.5-1.4)\end{array}$ \\
\hline $\begin{array}{c}\text { Some college (no degree) or } \\
\text { associates degree }\end{array}$ & 835 & 55.0 & 1.39 & 466 & 30.7 & 1.32 & $\begin{array}{c}0.8 \\
(0.6-0.9) \\
\end{array}$ & $\begin{array}{c}0.7 \\
(0.6-1.9)\end{array}$ & 111 & 7.1 & 0.71 & $\begin{array}{c}1.4 \\
(0.9-2.4)\end{array}$ & $\begin{array}{c}1.2 \\
(0.7-2.0) \\
\end{array}$ & 111 & 7.2 & 0.81 & $\begin{array}{c}1.3 \\
(0.8-2.2) \\
\end{array}$ & $\begin{array}{c}1.1 \\
(0.7-1.9)\end{array}$ \\
\hline Bachelor's degree or more & 220 & 51.3 & 2.89 & 122 & 29.5 & 2.54 & $\begin{array}{c}0.7 \\
(0.5-0.9) \\
\end{array}$ & $\begin{array}{c}0.6 \\
(0.5-0.9) \\
\end{array}$ & 35 & 8.8 & 1.35 & $\begin{array}{c}1.7 \\
(0.9-3.2) \\
\end{array}$ & $\begin{array}{c}1.1 \\
(0.5-2.2)\end{array}$ & 40 & 10.4 & 1.59 & $\begin{array}{c}1.8 \\
(1.1-3.1) \\
\end{array}$ & $\begin{array}{c}1.1 \\
(0.6-2.1) \\
\end{array}$ \\
\hline \multicolumn{19}{|l|}{ Poverty level } \\
\hline Below poverty level & 935 & 57.8 & 1.21 & 508 & 32.5 & 1.29 & ref & ref & 78 & 4.6 & 0.52 & ref & ref & 81 & 5.1 & 0.59 & ref & ref \\
\hline
\end{tabular}


Table 4. Cont.

\begin{tabular}{|c|c|c|c|c|c|c|c|c|c|c|c|c|c|c|c|c|c|c|}
\hline & \multicolumn{18}{|c|}{ Transitions assessed between Wave 1 \& Wave 2 (W2) } \\
\hline & \multicolumn{3}{|c|}{$\begin{array}{l}\text { No transition a } \\
\text { (i.e., poly cigarette use } \\
\text { at W2; referent group) }\end{array}$} & \multicolumn{5}{|c|}{$\begin{array}{l}\text { Transition to exclusive cigarette } \\
\text { (i.e., cigarette use at } \mathrm{W} 2 \text {, no use of any other } \\
\text { tobacco product at } \mathrm{W} 2 \text { ) }\end{array}$} & \multicolumn{5}{|c|}{$\begin{array}{l}\text { Transition to non-cigarette tobacco use } \\
\text { (i.e., no cigarette use at W2, but use of any other } \\
\text { tobacco product(s) at W2; } 56.4 \% \text { of people in this } \\
\text { transition group were ENDS users at W2) }\end{array}$} & \multicolumn{5}{|c|}{$\begin{array}{l}\text { Discontinue tobacco use } \\
\text { (i.e., no use of any tobacco product at W2) }\end{array}$} \\
\hline & & & & & & & $\begin{array}{l}\text { Model } 1{ }^{1} \\
\end{array}$ & Model $2^{2}$ & & & & Model $1{ }^{1}$ & Model $2^{2}$ & & & & $\begin{array}{l}\text { Model } 1^{1} \\
\end{array}$ & Model 22 \\
\hline At or near poverty level & 529 & 55.5 & 1.63 & 291 & 30.8 & 1.49 & $\begin{array}{c}1.1 \\
(0.9-1.3)\end{array}$ & $\begin{array}{c}1.0 \\
(0.8-1.3)\end{array}$ & 75 & 7.3 & 0.95 & $\begin{array}{c}1.6 \\
(1.1-2.3) \\
\end{array}$ & $\begin{array}{c}1.5 \\
(1.0-2.3) \\
\end{array}$ & 57 & 6.4 & 0.93 & $\begin{array}{c}1.4 \\
(0.9-2.1) \\
\end{array}$ & $\begin{array}{c}1.3 \\
(0.8-2.1) \\
\end{array}$ \\
\hline $\begin{array}{l}\text { At or above twice the } \\
\text { poverty level }\end{array}$ & 575 & 49.1 & 1.64 & 374 & 34.9 & 1.78 & $\begin{array}{c}1.4 \\
(1.2-1.8)\end{array}$ & $\begin{array}{c}1.5 \\
(1.2-1.8)\end{array}$ & 93 & 8.9 & 1.14 & $\begin{array}{c}2.1 \\
(1.4-3.2)\end{array}$ & $\begin{array}{c}2.0 \\
(1.3-3.1)\end{array}$ & 82 & 7.1 & 0.91 & $\begin{array}{c}1.7 \\
(1.1-2.5)\end{array}$ & $\begin{array}{c}1.5 \\
(0.9-2.3) \\
\end{array}$ \\
\hline Not reported & 156 & 58.5 & 3.93 & 75 & 27.7 & 3.53 & $\begin{array}{c}0.8 \\
(0.5-1.3)\end{array}$ & $\begin{array}{c}0.8 \\
(0.5-1.2)\end{array}$ & 15 & 5.5 & 1.49 & $\begin{array}{c}1.0 \\
(0.5-2.2)\end{array}$ & $\begin{array}{c}1.3 \\
(0.6-2.7)\end{array}$ & 22 & 8.3 & 1.97 & $\begin{array}{c}1.4 \\
(0.8-2.7)\end{array}$ & $\begin{array}{c}1.5 \\
(0.7-3.0) \\
\end{array}$ \\
\hline \multicolumn{19}{|l|}{$\begin{array}{c}\text { Total number of products } \\
\text { currently used }\end{array}$} \\
\hline 2 products & 1191 & 48.1 & 1.15 & 934 & 38.9 & 1.17 & ref & ref & 146 & 6.2 & 0.64 & ref & ref & 160 & 6.8 & 0.64 & ref & ref \\
\hline 3 or more products & 927 & 67.6 & 1.40 & 259 & 19.3 & 1.25 & $\begin{array}{c}0.4 \\
(0.3-0.5)\end{array}$ & $\begin{array}{c}0.6 \\
(0.4-0.8)\end{array}$ & 105 & 7.8 & 0.84 & $\begin{array}{c}0.9 \\
(0.6-1.3) \\
\end{array}$ & $\begin{array}{c}1.0 \\
(0.5-2.1)\end{array}$ & 75 & 5.3 & 0.61 & $\begin{array}{c}0.5 \\
(0.4-0.8) \\
\end{array}$ & $\begin{array}{c}0.5 \\
(0.2-1.0) \\
\end{array}$ \\
\hline \multicolumn{19}{|l|}{ Nicotine dependence ${ }^{3}$} \\
\hline 1st quartile & 539 & 50.5 & 1.82 & 288 & 28.2 & 1.63 & ref & ref & 99 & 10.3 & 1.17 & ref & ref & 112 & 11.1 & 1.16 & ref & ref \\
\hline 2nd quartile & 496 & 53.1 & 1.75 & 290 & 33.3 & 1.64 & $\begin{array}{c}1.1 \\
(0.9-1.4)\end{array}$ & $\begin{array}{c}1.2 \\
(0.9-1.5)\end{array}$ & 71 & 7.5 & 1.07 & $\begin{array}{c}0.7 \\
(0.5-1.1)\end{array}$ & $\begin{array}{c}0.9 \\
(0.6-1.4)\end{array}$ & 53 & 6.2 & 0.89 & $\begin{array}{c}0.6 \\
(0.4-0.9)\end{array}$ & $\begin{array}{c}0.9 \\
(0.6-1.4)\end{array}$ \\
\hline 3rd quartile & 605 & 59.0 & 1.76 & 322 & 32.0 & 1.61 & $\begin{array}{c}1.0 \\
(0.8-1.2)\end{array}$ & $\begin{array}{c}1.1 \\
(0.8-1.4)\end{array}$ & 46 & 4.4 & 0.70 & $\begin{array}{c}0.4 \\
(0.3-0.6)\end{array}$ & $\begin{array}{c}0.6 \\
(0.4-1.0)\end{array}$ & 42 & 4.6 & 0.84 & $\begin{array}{c}0.4 \\
(0.2-0.7)\end{array}$ & $\begin{array}{c}0.7 \\
(0.4-1.2) \\
\end{array}$ \\
\hline 4th quartile & 532 & 55.5 & 1.80 & 332 & 36.8 & 1.83 & $\begin{array}{c}1.1 \\
(0.8-1.4)\end{array}$ & $\begin{array}{c}1.2 \\
(0.9-1.6)\end{array}$ & 43 & 4.7 & 0.72 & $\begin{array}{c}0.5 \\
(0.3-0.8) \\
\end{array}$ & $\begin{array}{c}0.7 \\
(0.4-1.3)\end{array}$ & 32 & 3.1 & 0.55 & $\begin{array}{c}0.3 \\
(0.2-0.5)\end{array}$ & $\begin{array}{c}0.5 \\
(0.3-1.0) \\
\end{array}$ \\
\hline \multicolumn{19}{|l|}{ Current use of cigarettes } \\
\hline Someday use & 458 & 49.0 & 1.84 & 199 & 23.1 & 1.65 & ref & ref & 129 & 14.6 & 1.36 & ref & ref & 119 & 13.3 & 1.31 & ref & ref \\
\hline Daily use & 1737 & 56.1 & 1.09 & 1049 & 35.2 & 1.04 & $\begin{array}{c}1.3 \\
(1.0-1.7)\end{array}$ & $\begin{array}{c}1.0 \\
(0.8-1.4)\end{array}$ & 132 & 4.4 & 0.42 & $\begin{array}{c}0.3 \\
(0.2-0.4) \\
\end{array}$ & $\begin{array}{c}0.4 \\
(0.2-0.5) \\
\end{array}$ & 123 & 4.2 & 0.43 & $\begin{array}{c}0.3 \\
(0.2-0.4)\end{array}$ & $\begin{array}{c}0.3 \\
(0.2-0.5) \\
\end{array}$ \\
\hline \multicolumn{19}{|l|}{ Current use of ENDS } \\
\hline No use & 1053 & 54.1 & 1.34 & 595 & 31.8 & 1.24 & ref & ref & 138 & 7.3 & 0.72 & ref & ref & 133 & 6.7 & 0.71 & ref & ref \\
\hline Someday use & 942 & 53.0 & 1.43 & 596 & 35.8 & 1.26 & $\begin{array}{c}1.0 \\
(0.8-1.1) \\
\end{array}$ & $\begin{array}{c}0.8 \\
(0.6-1.1) \\
\end{array}$ & 84 & 5.0 & 0.59 & $\begin{array}{c}0.7 \\
(0.5-1.0) \\
\end{array}$ & $\begin{array}{c}0.8 \\
(0.5-1.2) \\
\end{array}$ & 97 & 6.2 & 0.64 & $\begin{array}{c}0.8 \\
(0.5-1.1) \\
\end{array}$ & $\begin{array}{c}1.0 \\
(0.6-1.7)\end{array}$ \\
\hline Daily use & 197 & 65.3 & 2.98 & 54 & 18.2 & 2.66 & $\begin{array}{c}0.4 \\
(0.3-0.6) \\
\end{array}$ & $\begin{array}{c}0.3 \\
(0.2-0.5) \\
\end{array}$ & 39 & 12.6 & 2.06 & $\begin{array}{c}1.3 \\
(0.8-2.2) \\
\end{array}$ & $\begin{array}{c}1.3 \\
(0.7-2.2) \\
\end{array}$ & 12 & 3.9 & 1.06 & $\begin{array}{c}0.4 \\
(0.2-0.8) \\
\end{array}$ & $\begin{array}{c}0.5 \\
(0.2-1.0) \\
\end{array}$ \\
\hline \multicolumn{19}{|l|}{$\begin{array}{c}\text { Current use of traditional } \\
\text { cigars/cigarillos/filtered } \\
\text { cigars }\end{array}$} \\
\hline No use & 941 & $\begin{array}{ll}49.4 \\
\end{array}$ & 1.42 & 680 & 36.9 & 1.34 & ref & ref & 124 & 6.9 & 0.69 & ref & ref & 120 & 6.8 & 0.63 & ref & ref \\
\hline Someday use & 1047 & 59.5 & 1.38 & 476 & 28.9 & 1.22 & $\begin{array}{c}0.7 \\
(0.6-0.9) \\
\end{array}$ & $\begin{array}{c}0.7 \\
(0.5-1.0) \\
\end{array}$ & 106 & 6.0 & 0.66 & $\begin{array}{c}0.7 \\
(0.5-1.0) \\
\end{array}$ & $\begin{array}{c}0.7 \\
(0.4-1.2)\end{array}$ & 98 & 5.7 & 0.64 & $\begin{array}{c}0.8 \\
(0.6-1.1)\end{array}$ & $\begin{array}{c}1.0 \\
(0.6-1.7) \\
\end{array}$ \\
\hline Daily use & 170 & 62.2 & 3.23 & 58 & 20.5 & 2.47 & $\begin{array}{c}0.4 \\
(0.3-0.6) \\
\end{array}$ & $\begin{array}{c}0.3 \\
(0.2-0.5) \\
\end{array}$ & 26 & 10.5 & 2.30 & $\begin{array}{c}1.4 \\
(0.8-2.6) \\
\end{array}$ & $\begin{array}{c}1.6 \\
(0.8-3.5) \\
\end{array}$ & 20 & 6.7 & 1.52 & $\begin{array}{c}1.0 \\
(0.6-1.8) \\
\end{array}$ & $\begin{array}{c}1.2 \\
(0.5-2.6) \\
\end{array}$ \\
\hline \multicolumn{19}{|l|}{ Current use of pipe } \\
\hline No use & 1993 & 53.7 & 0.97 & 1182 & 33.3 & 0.95 & ref & ref & 237 & 6.5 & 0.50 & ref & ref & 230 & 6.4 & $\begin{array}{ll}0.48 \\
\end{array}$ & ref & ref \\
\hline
\end{tabular}


Table 4. Cont.

\begin{tabular}{|c|c|c|c|c|c|c|c|c|c|c|c|c|c|c|c|c|c|c|}
\hline & \multicolumn{18}{|c|}{ Transitions assessed between Wave 1 \& Wave 2 (W2) } \\
\hline & \multicolumn{3}{|c|}{$\begin{array}{l}\text { No transition a } \\
\text { (i.e., poly cigarette use } \\
\text { at W2; referent group) }\end{array}$} & \multicolumn{5}{|c|}{$\begin{array}{l}\text { Transition to exclusive cigarette } \\
\text { (i.e., cigarette use at } \mathrm{W} 2 \text {, no use of any other } \\
\text { tobacco product at } \mathrm{W} 2 \text { ) }\end{array}$} & \multicolumn{5}{|c|}{$\begin{array}{l}\text { Transition to non-cigarette tobacco use } \\
\text { (i.e., no cigarette use at } \mathrm{W} 2 \text {, but use of any other } \\
\text { tobacco product(s) at } \mathrm{W} 2 ; 56.4 \% \text { of people in this } \\
\text { transition group were ENDS users at } W 2 \text { ) }\end{array}$} & \multicolumn{5}{|c|}{$\begin{array}{l}\quad \text { Discontinue tobacco use } \\
\text { (i.e., no use of any tobacco product at W2) }\end{array}$} \\
\hline & & & & & & & Model 1 ${ }^{1}$ & Model 2 ${ }^{2}$ & & & & Model $1^{1}$ & Model $2^{2}$ & & & & Model $1{ }^{1}$ & ${\text { Model } 2^{2}}^{2}$ \\
\hline Someday use & 193 & 64.9 & 3.13 & 61 & 21.8 & 2.98 & $\begin{array}{c}0.7 \\
(0.5-1.0)\end{array}$ & $\begin{array}{c}1.0 \\
(0.6-1.6)\end{array}$ & 22 & 8.6 & 2.28 & $\begin{array}{c}1.1 \\
(0.6-2.1)\end{array}$ & $\begin{array}{c}1.5 \\
(0.8-2.7)\end{array}$ & 11 & 4.6 & 1.53 & $\begin{array}{c}0.8 \\
(0.4-1.7) \\
\end{array}$ & $\begin{array}{c}1.1 \\
(0.4-2.9)\end{array}$ \\
\hline Daily use & 6 & 41.9 & 12.85 & 4 & 34.0 & 14.13 & $\begin{array}{c}1.7 \\
(0.4-6.8)\end{array}$ & $\begin{array}{c}1.4 \\
(0.2-10.9) \\
\end{array}$ & 2 & 17.7 & 10.33 & $\begin{array}{c}3.5 \\
(0.6-19.6) \\
\end{array}$ & $\begin{array}{c}3.2 \\
(0.5-20.1) \\
\end{array}$ & 1 & 6.4 & 7.72 & $\begin{array}{c}2.0 \\
(0.1-41.3) \\
\end{array}$ & $\begin{array}{c}2.3 \\
(0.1-68.7) \\
\end{array}$ \\
\hline \multicolumn{19}{|c|}{ Current use of hookah } \\
\hline No use & 1590 & 53.7 & 0.96 & 991 & 34.3 & 0.94 & ref & ref & 180 & 6.4 & 0.56 & ref & ref & 156 & 5.6 & 0.52 & ref & ref \\
\hline Someday use & 590 & 57.5 & 2.02 & 254 & 26.5 & 1.81 & $\begin{array}{c}0.9 \\
(0.7-1.1) \\
\end{array}$ & $\begin{array}{c}1.0 \\
(0.7-1.4)\end{array}$ & 79 & 7.6 & 0.83 & $\begin{array}{c}1.1 \\
(0.8-1.6)\end{array}$ & $\begin{array}{c}1.1 \\
(0.6-1.8)\end{array}$ & 82 & 8.3 & 1.05 & $\begin{array}{c}1.1 \\
(0.7-1.7)\end{array}$ & $\begin{array}{c}1.4 \\
(0.8-2.5) \\
\end{array}$ \\
\hline Daily use & 14 & 54.2 & 13.75 & 1 & 8.8 & 9.20 & $\begin{array}{c}0.3 \\
(0.0-4.7) \\
\end{array}$ & $\begin{array}{c}0.6 \\
(0.0-7.6) \\
\end{array}$ & 2 & 17.6 & 11.99 & $\begin{array}{c}3.3 \\
(0.4-26.4) \\
\end{array}$ & $\begin{array}{c}4.2 \\
(0.5-34.8) \\
\end{array}$ & 3 & 19.4 & 11.39 & $\begin{array}{c}3.0 \\
(0.5-17.8) \\
\end{array}$ & $\begin{array}{c}5.1 \\
(0.4-62.6) \\
\end{array}$ \\
\hline \multicolumn{19}{|c|}{$\begin{array}{l}\text { Current use of smokeless/ } \\
\text { snus pouches/dissolvable }\end{array}$} \\
\hline No use & 1674 & 51.2 & 1.05 & 1125 & 36.0 & 1.00 & ref & ref & 189 & 6.0 & 0.52 & ref & ref & 211 & 6.8 & $\begin{array}{l}0.54 \\
\end{array}$ & ref & ref \\
\hline Someday use & 345 & 68.7 & 2.28 & 99 & 21.9 & 1.99 & $\begin{array}{c}0.5 \\
(0.4-0.7)\end{array}$ & $\begin{array}{c}0.6 \\
(0.4-0.8)\end{array}$ & 27 & 5.2 & 1.44 & $\begin{array}{c}0.6 \\
(0.3-1.1)\end{array}$ & $\begin{array}{c}0.6 \\
(0.3-1.3)\end{array}$ & 20 & 4.2 & 0.94 & $\begin{array}{c}0.6 \\
(0.3-1.1)\end{array}$ & $\begin{array}{c}0.8 \\
(0.4-1.6)\end{array}$ \\
\hline Daily use & 142 & 71.6 & 3.54 & 7 & 4.0 & 1.37 & $\begin{array}{c}0.1 \\
(0.0-0.2)\end{array}$ & $\begin{array}{c}0.1 \\
(0.0-0.1)\end{array}$ & 39 & 20.2 & 3.25 & $\begin{array}{c}2.1 \\
(1.2-3.7)\end{array}$ & $\begin{array}{c}1.8 \\
(0.9-3.3)\end{array}$ & 8 & 4.2 & 1.72 & $\begin{array}{c}0.6 \\
(0.2-1.8)\end{array}$ & $\begin{array}{c}0.6 \\
(0.2-2.0)\end{array}$ \\
\hline
\end{tabular}

1 Table 4 notes. Sample includes adults aged 18+ years who were 'poly cigarette' smokers at W1 and also participated in W2 (N = 3946); analyses weighted using W2 longitudinal weights. Results from multinomial logistic regression analyses indicate the relative probability of being in each W2 transition group vs the W2 'no transition' group as a function of correlates assessed at W1. Current use at each wave defined for cigarettes as having smoked at least 100 cigarettes in lifetime and now smoking everyday or somedays; for hookah as now using everyday, somedays, or monthly; for all other products as now using everyday or somedays. 'Poly cigarette' use defined as current use of cigarettes and current use of at least one other tobacco product. 'Exclusive cigarette' use defined as current use of cigarettes and no current use of any other tobacco product. 'Non-cigarette tobacco' use defined as no current use of cigarettes and current use of any other tobacco product. Bold font indicates significant association at $\alpha=0.05$; grey shaded cells indicate unstable estimate due to denominator $<50$ or relative standard of the estimate $>30 \% .{ }^{1}$ Analyses adjusted for age, sex, sexual orientation, race/ethnicity, education, and poverty level. ${ }^{2}$ Analyses adjusted for age, sex, sexual orientation, race/ethnicity, education, poverty level, total number of products used, nicotine dependence, and type and frequency of product used. ${ }^{3}$ Mean cumulative nicotine dependence score quartiles determined among W1 'poly cigarette' smokers. ${ }^{a}$ No transition is defined relative to 'poly cigarette' use; therefore, no transition may reflect the same or different combinations of products used at W2 as were used at W1. 


\section{Discussion}

Data from W1 and W2 of the PATH Study indicate that transitions in tobacco product use among adult tobacco users, particularly young adults, are common, and that behaviors vary more and in different ways for different demographic groups and dependence levels, suggesting differing needs for tobacco control efforts among subgroups of the population. Among exclusive cigarette smokers, male smokers and those not identifying as heterosexual/straight were more likely to add other combustible tobacco product use to their cigarette use; female smokers, white smokers, and those not identifying as heterosexual/straight were more likely to add ENDS use to their cigarette use; and exclusive cigarette smokers below the poverty level were less likely to discontinue tobacco use than were their counterparts at or above twice the poverty level. Among poly-cigarette smokers, female smokers, those with higher education, and those with higher income were more likely to transition to exclusive cigarette use than their counterparts; and female smokers, and Hispanic smokers (compared to non-Hispanic white smokers), were more likely to discontinue tobacco use. These initial demographic findings can serve as a foundation for additional work that can help determine what drives demographic differences in tobacco use transitions. For example, differences in rates of exclusive cigarette smokers adding ENDS use found here may reflect demographic differences in quit intentions, as others have found that cigarette smokers report using ENDS to try to quit smoking $[24,25]$.

Further, nicotine dependence remains a key barrier to discontinuing tobacco use. These findings show that those using tobacco on a daily basis and scoring higher on an index of nicotine dependence [20] were less likely to discontinue cigarette use and all tobacco use than their counterparts. Importantly, tobacco users with higher dependence scores were more likely to switch among tobacco products, and relatively highly dependent cigarette smokers were more likely to add product(s) to their cigarette use compared to their less dependent counterparts. Further research can elucidate these findings, but one possible explanation is that highly dependent cigarette smokers may be turning to other tobacco products as a means of trying to reduce or stop their cigarette use [24]. Limiting the amount of nicotine contained in cigarettes to reduce their addictiveness [26] has potential to mitigate the disease burden caused by combusted cigarettes, a high public health priority [27].

\section{Future Directions}

These PATH Study findings provide a broad look at demographic and tobacco use characteristics associated with transitions in tobacco product use among current tobacco users over a 12-month period, and longer follow up can help us to better understand the effects of these transitions on public health. Additionally, as the variety of ENDS products available to consumers today expands, considering different types of ENDS products rather than considering all ENDS as one product could be useful for understanding transitions [3]. The PATH Study data can also be used to evaluate correlates of transitions among non-tobacco users (i.e., experimentation and initiation of tobacco use and relapse among former tobacco users), which will be important for determining the public health implications of the diverse tobacco product landscape. The work we present here can also be extended by evaluating changes in frequency of use within and among tobacco product types and further disaggregating transitions among poly-tobacco product users, all in an effort to help inform tobacco regulatory efforts aimed at protecting the health of the U.S. population.

\section{Conclusions}

Transitions in tobacco product use among adult tobacco users in the U.S. vary among different demographic groups, suggesting differing needs for tobacco control efforts among subgroups of the population. High nicotine dependence predicts transitioning among or the addition of tobacco products and is a barrier to discontinuing tobacco use, adding evidence to support policy to lower nicotine content of cigarettes and to evaluate new products for their potential to reduce cigarette use. 
Author Contributions: A.J.H., K.A.K., N.B., W.M.C. conceived the study. All authors contributed to the design of the study. K.A.K., E.S. conducted the data analysis. All authors contributed to the interpretation of results. K.A.K. wrote the first draft of the manuscript. All authors critically revised the manuscript and approved the final version.

Funding: This manuscript is supported with Federal funds from the National Institute on Drug Abuse, National Institutes of Health, and the Center for Tobacco Products, Food and Drug Administration, Department of Health and Human Services, under a contract to Westat (Contract No. HHSN271201100027C).

Acknowledgments: The views and opinions expressed in this manuscript are those of the authors only and do not necessarily represent the views, official policy or position of the U.S. Department of Health and Human Services or any of its affiliated institutions or agencies.

Conflicts of Interest: Compton reports long-term stock holdings in General Electric, the 3M Companies, and Pfizer Incorporated, unrelated to this manuscript; Cummings has received grant funding from Pfizer, Inc., to study the impact of a hospital based tobacco cessation intervention. Cummings also receives funding as an expert witness in litigation filed against the tobacco industry; Goniewicz receives fees for serving on an advisory board from Johnson \& Johnson and grant support from Pfizer outside of the submitted work; Niaura reports having been a witness for plaintiffs vs. tobacco companies, receiving speaker fees, receiving honoraria, sitting on advisory boards, being a site PI, and consulting for pharmaceutical companies testing and marketing smoking cessation aids, but not in the last 6 years. Fong has a Senior Investigator Award from the Ontario Institute for Cancer Research and Prevention Scientist Award from the Canadian Cancer Society Research Institute. Conway reports preparing this article while employed at the NIH/National Institute on Drug Abuse. No other potential conflict of interest relevant to this manuscript was reported.

\section{References}

1. O'Connor, R.J. Non-cigarette tobacco products: What have we learnt and where are we headed? Tob. Control 2012, 21, 181-190. [CrossRef] [PubMed]

2. Zhu, S.; Sun, J.Y.; Bonnevie, E.; Cummins, S.E.; Gamst, A.; Yin, L.; Lee, M. Four hundred and sixty brands of e-cigarettes and counting: Implications for product regulation. Tob. Control 2014, 23, iii3-iii9. [CrossRef] [PubMed]

3. Balbao, S.; Stepanov, I. The wild west of e-cigarettes. Chem. Res. Toxicol. 2018, 31, 823-824. [CrossRef] [PubMed]

4. Agaku, I.T.; King, B.A.; Husten, C.G.; Bunnell, R.; Ambrose, B.K.; Hu, S.; Holder-Hayes, E.; Day, H.R. Tobacco product use among adults-United States, 2012-2013. Morb. Mortal. Wkly. Rep. 2014, 63, 542-547.

5. Cohn, A.; Ehlke, S.; Cobb, C.O.; Soule, E.K. Hookah tobacco smoking in a large urban sample of adult cigarette smokers: Links with alcohol and poly-tobacco use. Addict. Behav. 2017, 68, 1-5. [CrossRef] [PubMed]

6. King, B.A.; Patel, R.; Nguyen, K.; Dube, S.R. Trends in awareness and use of electronic cigarettes among U.S. adults, 2010-2013. Nicotine Tob. Res. 2015, 17, 219-227. [CrossRef] [PubMed]

7. Kasza, K.A.; Borek, B.; Conway, K.P.; Goniewicz, M.L.; Stanton, C.A.; Sharma, E.; Fong, G.T.; Abrams, D.B.; Coleman, B.; Schneller, L.M.; et al. Transitions in Tobacco Product Use by U.S. Adults between 2013-14 and 2014-15: Findings from the PATH Study Wave 1 and Wave 2. Int. J. Environ. Res. Public Health 2018, 15, 2515. [CrossRef]

8. Tam, J.; Day, H.R.; Rostron, B.L.; Apelberg, B.J. A systematic review of transitions between cigarette and smokeless tobacco product use in the United States. BMC Public Health 2015, 15, 258. [CrossRef] [PubMed]

9. Bondy, S.J.; Victor, C.; Diemert, L.M.; Mecredy, G.C.; Chaiton, M.; Brown, K.S.; Cohen, J.E.; McDonald, P.W.; Ferrence, R.; Garcia, J.M.; et al. Transitions in Smoking Status Over Time in a Population-Based Panel Study of Smokers. Nicotine Tob. Res. 2013, 15, 1201-1210. [CrossRef] [PubMed]

10. Barbeau, E.M.; Leavy-Sperounis, A.; Balbach, E.D. Smoking, social class, and gender: What can public health learn from the tobacco industry about disparities in smoking? Tob. Control 2004, 13, 115-120. [CrossRef] [PubMed]

11. Blosnich, J.R.; Jarrett, T.; Horn, K. Racial and ethnic differences in current use of cigarettes, cigars, and hookahs among lesbian, gay, and bisexual young adults. Nicotine Tob. Res. 2011, 13, 487-491. [CrossRef] [PubMed]

12. Giovino, G.A. Epidemiology of tobacco use in the United States. Oncogene 2002, 21, 7326-7340. [CrossRef] [PubMed] 
13. Fagan, P.; Moolchan, E.T.; Lawrence, D.; Fernander, A.; Ponder, P.K. Identifying health disparities across the tobacco continuum. Addiction 2007, 102, 5-29. [CrossRef] [PubMed]

14. Institute of Medicine. The Health of Lesbian, Gay, Bisexual, and Transgender People: Building a Foundation for Better Understanding; Institute of Medicine: Washington, DC, USA, 2001.

15. U.S. Department of Health and Human Services. The Health Consequences of Smoking - 50 Years of Progress: A Report of the Surgeon General; U.S. Department of Health and Human Services, Centers for Disease Control and Prevention, National Center for Chronic Disease Prevention and Health Promotion, Office on Smoking and Health: Atlanta, GA, USA, 2014.

16. U.S. Food \& Drug Administration. FDA News Release: FDA Announces Comprehensive Regulatory Plan to Shift Trajectory of Tobacco-Related Disease, Death. Available online: https:/ / www.fda.gov/NewsEvents/ Newsroom/PressAnnouncements/ucm568923.htm (accessed on 28 July 2017).

17. Hyland, A.; Ambrose, B.K.; Conway, K.P.; Borek, N.; Lambert, E.; Carusi, C.; Taylor, K.; Crosse, S.; Fong, G.T.; Cummings, K.M.; et al. Design and methods of the Population Assessment of Tobacco and Health (PATH) Study. Tob. Control 2017, 26, 371-378. [CrossRef] [PubMed]

18. Population Assessment of Tobacco and Health (PATH) Study. Nonresponse Bias Analysis. Available online: http:/ / doi.org/10.3886/ICPSR36231 (accessed on 1 July 2016).

19. Population Assessment of Tobacco and Health (PATH) Study. Available online: https://doi.org/10.3886/ Series606 (accessed on 1 July 2016).

20. Strong, D.R.; Pearson, J.; Ehlkeb, S.; Kirchner, T.; Abrams, D.; Taylor, K.; Compton, W.M.; Conway, K.P.; Lambert, E.; Green, V.R.; et al. Indicators of dependence for different types of tobacco product users: Descriptive findings from Wave 1 (2013-2014) of the Population Assessment of Tobacco and Health (PATH) study. Drug Alcohol Depend. 2017, 178, 257-266. [CrossRef] [PubMed]

21. StataCorp. Stata Statistical Software: Release 14; StataCorp LP: College Station, TX, USA, 2011.

22. McCarthy, P.J. Pseudoreplication: Further evaluation and applications of the balanced half-sample technique. Vital Health Stat. 1969, 2, 1-24.

23. Judkins, D.R. Fay's method for variance estimation. J. Off. Stat. 1990, 6, 223-239.

24. Richardson, A.; Pearson, J.; Xiao, H.; Stalgaitis, C.; Vallone, D. Prevalence, harm perceptions, and reasons for using noncombustible tobacco products among current and former smokers. Am. J. Public Health 2014, 104, 1437-1444. [CrossRef] [PubMed]

25. Delnevo, C.D.; Giovenco, D.P.; Steinberg, M.B.; Villanti, A.C.; Pearson, J.L.; Niaura, R.S.; Abrams, D.B. Patterns of electronic cigarette use among adults in the United States. Nicotine Tob. Res. 2016, 18, 715-719. [CrossRef] [PubMed]

26. U.S. Department of Health and Human Services. How Tobacco Smoke Causes Disease: The Biology and Behavioral Basis for Smoking-Attributable Disease: A Report of the Surgeon General; U.S. Department of Health and Human Services, Centers for Disease Control and Prevention, National Center for Chronic Disease Prevention and Health Promotion, Office on Smoking and Health: Atlanta, GA, USA, 2010.

27. Gottlieb, S.; Zeller, M. A Nicotine-Focused Framework for Public Health. N. Engl. J. Med. 2017, 377, 1111-1114. [CrossRef] [PubMed]

(C) 2018 by the authors. Licensee MDPI, Basel, Switzerland. This article is an open access article distributed under the terms and conditions of the Creative Commons Attribution (CC BY) license (http://creativecommons.org/licenses/by/4.0/). 